\title{
Impaired immune signaling and changes in the lung microbiome precede secondary bacterial pneumonia in COVID-19
}

\section{Alexandra Tsitsiklis}

University of California, San Francisco https://orcid.org/0000-0003-3467-1171

\section{Beth Zha}

University of California, San Francisco

\section{Ashley Byrne}

Chan Zuckerberg Biohub https://orcid.org/0000-0002-2177-924X

\section{Catherine DeVoe}

University of California, San Francisco

\section{Sophia Levan}

University of California, San Francisco

\section{Elze Rackaityte}

University of California, San Francisco

\section{Sara Sunshine}

University of California, San Francisco

\section{Eran Mick}

University of California, San Francisco https://orcid.org/0000-0002-7299-808X

\section{Rajani Ghale}

University of California, San Francisco

\section{Alejandra Jauregui}

University of California, San Francisco

\section{Norma Neff}

Chan Zuckerberg Biohub https://orcid.org/0000-0001-7141-5420

\section{Aartik Sarma}

University of California, San Francisco https://orcid.org/0000-0002-7508-7345

\section{Paula Serpa}

University of California, San Francisco

\section{Thomas Deiss}

University of California, San Francisco

\section{Amy Kistler}

Chan Zuckerberg Biohub

\section{Sidney Carrillo}


University of California, San Francisco

\section{K. Mark Ansel}

University of California, San Francisco https://orcid.org/0000-0003-4840-9879

\section{Aleksandra Leligdowicz}

University of California, San Francisco

\section{Stephanie Christenson}

University of California, San Francisco

\section{Norman Jones}

University of California, San Francisco

\section{Bing Wu}

Genentech, Inc

\section{Spyros Darmanis}

Genentech, Inc

\section{Michael Matthay}

University of California, San Francisco https://orcid.org/0000-0003-3039-8155

\section{Susan Lynch}

University of California, San Francisco Joseph DeRisi

University of California, San Francisco

\section{COMET Consortium}

University of California, San Francisco

\section{Carolyn Hendrickson}

University of California, San Francisco

\section{Kirsten Kangelaris}

University of California, San Francisco

\section{Matthew Krummel}

University of California, San Francisco

https://orcid.org/0000-0001-5695-7336

\section{Prescott Woodruff}

University of California, San Francisco

\section{David Erle}

University of California, San Francisco https://orcid.org/0000-0002-2171-0648

\section{Oren Rosenberg}

University of California, San Francisco https://orcid.org/0000-0002-5736-4388

\section{Carolyn Calfee}

University of California, San Francisco

Charles Langelier ( $\sim$ Chaz.Langelier@ucsf.edu )

University of California, San Francisco https://orcid.org/0000-0002-6708-4646 
Article

Keywords: COVID-19, SARS-CoV-2, secondary bacterial pneumonia, VAP, metagenomics, 41 scRNA-seq

Posted Date: April 23rd, 2021

DOl: https://doi.org/10.21203/rs.3.rs-380803/v1

License: (c) (i) This work is licensed under a Creative Commons Attribution 4.0 International License. Read Full License 
Title: Impaired immune signaling and changes in the lung microbiome precede secondary

2 bacterial pneumonia in COVID-19

4 Authors:

5 †Alexandra Tsitsiklis ${ }^{1},{ }^{\dagger}$ Beth Shoshana Zha ${ }^{2},{ }^{\dagger}$ Ashley Byrne ${ }^{3},{ }^{\dagger}$ Catherine Devoe ${ }^{1},{ }^{\text {\#Sophia Levan }}{ }^{4}$, $6{ }^{\#}$ Elze Rackaityte ${ }^{5}$, Sara Sunshine ${ }^{5}$, Eran Mick ${ }^{1,2,3}$, Rajani Ghale ${ }^{1,2}$, Alejandra Jauregui ${ }^{2}$, Aartik

7 Sarma $^{2}$, Norma Neff ${ }^{3}$, Paula Hayakawa Serpa ${ }^{1}$, Thomas J. Deiss ${ }^{2}$, Amy Kistler $^{3}$, Sidney Carrillo²,

8 K. Mark Ansel ${ }^{6,7}$, Aleksandra Leligdowicz ${ }^{2}$, Stephanie Christenson ${ }^{2}$, Norman Jones ${ }^{8}$, Bing $\mathrm{Wu}^{9}$,

9 Spyros Darmanis ${ }^{9}$, Michael A. Matthay ${ }^{2}$, Susan V. Lynch ${ }^{10,11}$, Joseph L. DeRisi ${ }^{3,5}$, COMET

10 Consortium+, Carolyn M. Hendrickson ${ }^{2}$, Kirsten N. Kangelaris ${ }^{4}$, Matthew F. Krummel ${ }^{12}$, Prescott

11 G. Woodruff ${ }^{2,7}$, David J. Erle ${ }^{4,13,14}$, Oren Rosenberg ${ }^{1}$, Carolyn S. Calfee ${ }^{2}$, ${ }^{*}$ Charles R. Langelier ${ }^{1,3}$

$13 \quad{ }^{\#}$ equal contributions

Affiliations:

$16{ }^{1}$ Department of Medicine, Division of Infectious Diseases, University of California San Francisco,

17 San Francisco, CA, USA

18 2Department of Medicine, Division of Pulmonary, Critical Care, Allergy and Sleep Medicine,

19 University of California San Francisco, San Francisco, CA, USA

$20{ }^{3}$ Chan Zuckerberg Biohub, San Francisco, CA, USA

$21{ }^{4}$ Department of Medicine, University of California San Francisco, San Francisco, CA, USA

$22{ }^{5}$ Department of Biochemistry and Biophysics, University of California San Francisco, San

23 Francisco, CA, USA

$24{ }^{6}$ Department of Microbiology and Immunology, University of California, San Francisco, CA, USA

$25{ }^{7}$ Sandler Asthma Basic Research Center, University of California, San Francisco, CA, USA

$26{ }^{8}$ Department of Experimental Medicine, University of California, San Francisco, CA, USA 
$27{ }^{9}$ Genentech, Inc. San Francisco, CA, USA.

$28{ }^{10}$ Department of Gastroenterology, University of California, San Francisco, CA, USA

$29{ }^{11}$ Benioff Center for Microbiome Medicine, University of California, San Francisco, CA, USA

$30{ }^{12}$ Department of Pathology, University of California, San Francisco, CA, USA

$31{ }^{13}$ Lung Biology Center, University of California, San Francisco, CA, USA

$32{ }^{14}$ UCSF CoLabs, University of California, San Francisco, CA, USA

$33{ }^{+}$COMET (COVID-19 Multiphenotyping for Effective Therapies) Consortium members are listed

34 in the Supplementary Appendix.

35

36

37 *Corresponding author

38 Email: chaz.langelier@ucsf.edu

39

40 Keywords: COVID-19, SARS-CoV-2, secondary bacterial pneumonia, VAP, metagenomics, 41 scRNA-seq

42 


\section{Abstract}

Secondary bacterial infections, including ventilator-associated pneumonia (VAP), lead to

45 worse clinical outcomes and increased mortality following viral respiratory infections including in

46 patients with coronavirus disease 2019 (COVID-19). Using a combination of tracheal aspirate

47 bulk and single-cell RNA sequencing (scRNA-seq) we assessed lower respiratory tract immune

48 responses and microbiome dynamics in 28 COVID-19 patients, 15 of whom developed VAP, and

49 eight critically ill uninfected controls. Two days before VAP onset we observed a transcriptional

50 signature of bacterial infection. Two weeks prior to VAP onset, following intubation, we observed

51 a striking impairment in immune signaling in COVID-19 patients who developed VAP. Longitudinal

52 metatranscriptomic analysis revealed disruption of lung microbiome community composition in

53 patients with VAP, providing a connection between dysregulated immune signaling and outgrowth

54 of opportunistic pathogens. These findings suggest that COVID-19 patients who develop VAP

55 have impaired antibacterial immune defense detectable weeks before secondary infection onset. 
Introduction

Secondary bacterial pneumonia results in significant morbidity and mortality in patients with viral lower respiratory tract infections $(\mathrm{LRTI})^{1}$. This problem was evident in the 1918 influenza pandemic during which the majority of deaths were ultimately attributed to secondary bacterial pneumonia ${ }^{2}$. SARS-CoV-2 infection, like influenza, confers an increased risk of late onset

61 secondary bacterial infection, often manifesting as ventilator-associated pneumonia (VAP) ${ }^{3}$.

62 Marked heterogeneity exists with respect to the risk of VAP in patients with coronavirus disease

632019 (COVID-19), with incidence ranging from 12-87\% between published cohort studies ${ }^{4-7}$.

64 The mechanisms underlying VAP susceptibility in COVID-19 remain unknown, and no

65 biomarkers yet exist to inform risk of VAP at the time of intubation. Animal models of influenza

66 may provide some insight, suggesting a role for interferon-mediated suppression of cytokines

67 essential for bacterial defense, including neutrophil recruitment, antimicrobial peptide production

68 and the Th17 response ${ }^{8-10}$. Few human immunoprofiling studies have been conducted in VAP

69 however, and none have been reported in a prospective cohort of COVID-19 patients.

Lower respiratory infections represent a dynamic relationship between pathogen, host

71 response and the lung microbiome ${ }^{11}$. Despite their interconnected roles, no studies to date have

72 simultaneously profiled host immune responses and lung microbiome dynamics in the context of

73 VAP. For instance, while prior work has described lung microbiome disruption in patients with

$74 \mathrm{VAP}^{11,12}$, the question of whether host immune responses following viral infection may contribute

75 to this dysbiosis, leading to subsequent infection, remains unanswered.

Given the marked heterogeneity in VAP incidence among patients with COVID-194-7, as

77 well as gaps in mechanistic understanding of secondary bacterial pneumonia, we sought to

78 assess the molecular determinants of VAP in the setting of SARS-CoV-2 infection. We employed

79 a systems biology approach involving immunoprofiling the host transcriptional response and

80 simultaneously assessing lung microbiome dynamics, using a combination of bulk and single cell

81 RNA sequencing and extensive clinical phenotyping. We observed a striking impairment in 
82 antibacterial immune signaling at the time of intubation, that correlated with disruption of the lung

83 microbiome, weeks before the onset of VAP.

\section{Results}

We conducted a prospective case-control study of adults requiring mechanical ventilation

87 for COVID-19 or for illnesses other than pneumonia. Of 84 patients with COVID-19 initially

88 enrolled, tracheal aspirate (TA) specimens from 28 patients met inclusion criteria for analysis

89 (Methods, Figure 1). In addition, eight critically ill patients from a second cohort (Study 2,

90 Methods) were included as controls. Patients were enrolled at one tertiary care hospital and one

91 safety net hospital in San Francisco, California under research protocols approved by the

92 University of California San Francisco Institutional Review Board (Methods). We collected TA

93 periodically following intubation and performed bulk and scRNA-seq (Methods).

Patients with VAP were adjudicated using the United States Centers for Disease Control

$95(C D C)$ definition ${ }^{13}$, including a requirement for a positive bacterial TA culture $(N=10)$. Patients who met CDC VAP criteria but had negative bacterial TA cultures were only included in a secondary

97 analysis $(\mathrm{N}=5)$. We defined onset of VAP as the first day a patient developed any of the criteria 98 used to meet the definition, in accordance with CDC guidance. Patients who did not meet the 99 CDC-NHSN criteria for VAP, and for whom there was no sustained clinical suspicion for bacterial 100 pneumonia during the admission, were adjudicated as No-VAP $(\mathrm{N}=13)$.

We compared lower respiratory tract host transcriptional responses between the VAP and

102 No-VAP groups at two time points. "Early" time point TA samples were collected a median of two 103 days post-intubation and 17 days before VAP onset (bulk RNA-seq analysis) or nine days before 104 VAP onset (scRNA-seq). "Late" time point samples were collected a median of two days before 105 VAP onset for both bulk and scRNA-seq analyses and compared against samples collected from 106 No-VAP patients at similar timepoints post-intubation (Figure 1, Table S1, Table S2). We 107 additionally evaluated eight intubated patients with non-pneumonia illnesses as controls at the 
"early" time-point. There were no significant differences between groups with respect to age, gender, race or ethnicity (Table S1, S2). In addition, there were no differences between groups with respect to in-hospital receipt of any immunosuppressant or antibiotics prior to sample collection (Table S3).

\section{COVID-19 VAP is associated with a transcriptional signature of bacterial infection two days} before VAP onset

We began by assessing the lower respiratory host transcriptional response two days preceding VAP onset in COVID-19 patients. Differential gene expression analysis was carried out on TA bulk RNA-seq data from five patients who developed VAP (samples collected a median of two days before VAP onset) and eight patients who did not develop VAP collected within a similar time frame after intubation (Table S1). We identified 436 differentially expressed genes at a False Discovery Rate (FDR) $<0.1$ (Figure 2A) and performed gene set enrichment analysis (GSEA) (Figure 2B). The patients who developed VAP exhibited upregulation of pathways related to antibacterial immune responses, such as neutrophil degranulation, toll-like receptor signaling, cytokine signaling, and antigen presentation (Figure 2B). Interferon alpha/beta signaling was the most upregulated pathway, suggesting prolonged viral infection in patients with VAP. Ingenuity pathway analysis (IPA) additionally predicted broad activation of upstream inflammatory cytokines in patients who developed VAP, in particular IFN $\alpha$ and IFN $\gamma$ (Figure 2C).

\section{COVID-19 patients who develop VAP have attenuated immune signaling two weeks before} VAP onset

Given our findings of a unique lower respiratory host transcriptional signature in the 48 hours preceding VAP onset, we next asked whether differences in host immune signaling might exist even earlier, two or more weeks before clinical diagnosis of VAP, and whether such differences might explain the increased susceptibility to secondary bacterial infection in these 
134 patients. We thus compared TA gene expression soon after the time of intubation between 135 patients who eventually developed VAP (samples collected a median of two days post-intubation, 13617 days before VAP onset, $n=4$ ) and patients who did not develop VAP (samples collected a 137 median of two days after intubation, $n=8)$ (Table 1). We identified 154 differentially expressed 138 genes at FDR <0.1. The COVID-19 patients who developed VAP had lower expression of several 139 genes with roles in innate immunity including IFI30, MMP2, TLR9, and DEFB124 (Figure 3A). 140 GSEA further revealed that patients who developed VAP had lower expression of pathways 141 related to antibacterial immune responses including neutrophil degranulation, toll-like receptor 142 signaling, IL-17 signaling, antigen presentation and complement pathways and higher expression 143 of IFN-alpha/beta signaling pathways, more than two weeks before the onset of VAP (Figure 3B). 144 Additionally, pathways related to adaptive immunity such as $\mathrm{T}$ and $\mathrm{B}$ cell receptor signaling were 145 also downregulated in patients who subsequently developed VAP (Figure 3B).

146 To gauge the degree of immune signaling suppression compared to controls, we 147 performed a similar analysis on critically ill intubated patients without infection (Figure 3C). 148 Relative to the control group, multiple antibacterial immune pathways were downregulated in 149 COVID-19 patients, with the greatest attenuation in the VAP group (Figure 3C). Upstream 150 regulator analysis identified impaired activation of diverse cytokines in those with VAP, while 151 IFNB1 was notably upregulated (Figure 3D). Several pro-inflammatory cytokines were 152 downregulated in both groups compared to the controls (Figure S1). We expanded the 153 comparison at the "early" time-point to include patients with culture-negative VAP (VAP: n=6, No154 VAP: $n=11$ ) and observed similar differences at the pathway level (Figure S2).

155 Given prior reports demonstrating correlation between SARS-CoV-2 viral load and 156 interferon related gene expression ${ }^{14}$ we next asked whether viral load differed between VAP and 157 No-VAP patients. No differences in SARS-CoV-2 qPCR or viral reads per million (rpM) in bulk 158 RNA-seq data were found in the days following intubation $(P=0.84$ (RNA-seq), $P=0.53$ (PCR), 159 Figure S3). We also considered the possibility that differences in the number of days of steroid 
exposure prior to sample collection might explain results, but found no differences $(P=0.343)$

161 (Table S1).

162

COVID-19 VAP is associated with impaired anti-bacterial immune signaling in monocytes, macrophages and neutrophils

To further understand the mechanism of early downregulation of key pathways involved

166 in antibacterial responses, we next asked whether this was driven by any one local immune cell 167 type. We performed scRNA-seq on TA specimens obtained early during disease course (median 168 of nine days before VAP) and enriched for immune cells using CD45 selection (Methods). 169 Clustering based upon cellular transcriptional signatures indicated that monocytes, macrophages 170 and neutrophils were the most abundant cell types (Figure 4A, S4A) and thus we focused 171 transcriptional assessment on these populations. A comparison of cell type proportions did not 172 reveal statistically significant differences in populations of mono/macs, neutrophils or $\mathrm{T}$ cells in 173 COVID-19 patients who subsequently developed VAP (Figure 4B).

174 COVID-19 patients who developed VAP had distinct cell type-specific transcriptional 175 signatures compared to those without VAP at this "early" post-intubation time-point (Figure 4, S5, 176 S6). With respect to mono/macs and neutrophils, we identified 532 and 693 differential expressed 177 genes, respectively, at FDR< 0.05. Several genes with key roles in innate immunity were 178 downregulated in both cell types in the COVID-19 patients who subsequently developed VAP 179 versus those who did not, including IL1Rn, ICAM1, NFKB2, and ITGAX in neutrophils, as well as 180 the neutrophil chemokines CXCL2 and CXCL8 in mono/macs (Figure 4C, 4F, S5). In addition, 181 similar to the bulk RNA-seq results demonstrating upregulation of type I IFN signaling at this time182 point in patients who developed VAP, we noted upregulation of several interferon-induced genes 183 including IFI27 and IFI30 in mono/macs, and IFI30, IFITM1, and IFITM3 in neutrophils (Figure 184 4C, F). 
IPA canonical pathway analysis of gene expression within each cluster revealed downregulation of several cytokine and innate immune signaling pathways in the patients who 187 later developed VAP at the "early" post-intubation time-point. In the mono/mac cluster, this 188 included downregulation of IL-1, IL-6, and iNOS signaling, as well as Th17 and TNFR2 signaling 189 (Figure 4D). Analysis of the neutrophil cluster also demonstrated attenuated IL-1, IL-6, and 190 TNFR2 signaling and NF-KB pathways (Figure 4G). COVID-19 patients who subsequently 191 developed VAP demonstrated upregulation of oxidative phosphorylation and glutathione 192 detoxification in the mono/mac subset, and interferon signaling, oxidative phosphorylation and 193 EIF2 signaling in the neutrophil cluster. Computational prediction of upstream cytokine activation by IPA revealed impaired activation of multiple pro-inflammatory cytokines in both the mono/macs and neutrophils in patients who developed VAP, including TNF, CXCL8, and IL1B, as well as downregulation of key factors important in monocyte to macrophage differentiation (CSF2, CSF3, PF4) (Figure 4E, H).

In the T cell population, we identified 1318 differentially expressed genes at FDR $<0.05$.

Genes associated with T cell recruitment, including CXCR6, ITGA1 and ITGA4, which have been shown to regulate localization and retention of $T$ cells in the lung during viral infection ${ }^{15,16}$, were downregulated in patients with VAP. Additionally, genes indicative of T cell activation (CD69, CD96, LAG3, ICOS, CD27), signaling (CD3, ZAP70, ITK, CD8A, CD8B), and effector functions (IFNG, GZMA, GZMB, KLRG1) were significantly downregulated in patients with VAP, suggesting

204 an impairment in T cell responses (Figure S6A). IPA revealed downregulation of signaling 205 pathways crucial for T cell recruitment, such as integrin signaling, and activation, such as CD28 206 signaling in helper T cells and phospholipase C signaling (Figure S6B).

\section{Temporal dynamics of the host response in COVID-19 patients who develop VAP}

210 in COVID-19 patients from the time of intubation to development of VAP by evaluating differential 
211 gene expression between COVID-19 VAP patients at the "early" time point (median of 17 days 212 before VAP onset, $\mathrm{n}=4$ ) versus "late" time point (median of two days before VAP onset, $\mathrm{n}=5$ ) by 213 bulk RNA-seq. We identified 2705 differentially expressed genes (FDR<0.1) and unsupervised

214 hierarchical clustering of the 50 most significant genes demonstrated clear separation of the two

215 time-points (Figure 5A). GSEA revealed that type I interferon signaling was notably 216 downregulated at the "late" time-point most immediately preceding VAP onset in comparison to 217 the "early" timepoint (Figure 5B); however, expression was still significantly higher than in the 218 No-VAP patients (Figure 2B). Several other immune signaling pathways were more highly 219 expressed at this "late" time-point, presumably reflecting activation of an antibacterial response 220 in the setting of bacterial pneumonia (Figure 5B). Consistent with this, upstream regulator 221 analysis indicated increased activation of several pro-inflammatory cytokines and decreased 222 IFN $\alpha$ and IFN- $\lambda$ signaling at the "late" versus "early" time-points (Figure 5C).

223 In contrast, comparing No-VAP patients at the "early" $(\mathrm{n}=8)$ versus "late" $(\mathrm{n}=8)$ time-points 224 yielded only two genes with a padj $<0.1$, both of which were interferon-stimulated genes $(R S A D 2$ 225 and CMPK2) downregulated at the "late" time-point, suggesting that while the host response was 226 relatively unchanged in these patients, the antiviral response attenuated over time. Indeed, GSEA 227 revealed that type I interferon signaling, and other antiviral immune pathways were downregulated 228 in the patients who did not develop VAP at the later time-point (Figure S7).

229 Next, we performed a similar comparison between the "early" and "late" time-points based 230 on scRNA-seq data from patients who developed VAP. Differential gene expression analysis on 231 these two populations identified 1368 differentially expressed genes (FDR<0.05) in the mono/mac 232 cluster, and 1028 in the neutrophil cluster. IPA revealed upregulation of antibacterial signaling 233 pathways at the later time-point, including signaling by several cytokines in the mono/mac cluster 234 (IL-17, IL-6, IL-1, TNF, IL-23, IFN) (Figure 5D-E), congruent with the bulk RNA-seq analysis. 235 Furthermore, we identified 1397 differentially expressed genes (FDR < 0.05) in the T cell cluster 236 between the two time-points and noted upregulation of signaling pathways indicative of an active 
237 T cell response ${ }^{17}$ (e.g. ERK/MAPK, Tec kinase, and phospholipase $\mathrm{C}$ ) in the days preceding VAP, 238 which was also in agreement with the bulk RNA-seq results (Figure S6C).

We further assessed dynamics of host immune responses between VAP and No-VAP patients by performing longitudinal analyses of key immune signaling pathways, including all

241 patients with available TA samples (VAP $n=7$, No-VAP $n=10$ ). Onset of VAP in these patients

242 ranged from 10-39 days post intubation, with a median of 25 days, and treatment with

243 immunosuppressants did not differ significantly between VAP and no-VAP patients $(p=0.304$,

244 Fisher's exact test). We calculated pathway Z-scores for each sample by averaging Z-scores for

245 the top 20 leading edge genes of each pathway (Methods). Early attenuation of immune signaling

246 in the VAP group was conspicuous, and this pattern eventually resolved later in disease course

247 by the time secondary bacterial infection became established (Figures $5 \mathrm{E}-\mathrm{H}$ ). We confirmed that

248 the observed differences between VAP and no-VAP patients were not driven by differences in

249 treatment with immunosuppressants by comparing pathway Z-scores in patients that received 250 immunosuppressants and those that did not at the early time-point regardless of VAP group 251 (Figure S8).

253 Lung microbiome disruption precedes VAP in COVID-19 patients

We hypothesized that the innate immune suppression in patients who developed VAP 255 would correlate with viral load. Using TA metatranscriptomics to assess the lower respiratory 256 microbiome, we evaluated longitudinal changes in SARS-CoV-2 abundance. Although no 257 difference was observed at the "early" timepoint (Figure S3), the trajectory of SARS-CoV-2 viral 258 load differed significantly in patients who developed VAP $(p=0.0058)$, although in both groups 259 decreased over time (Figure 6A). This result suggested that COVID-19 patients who develop 260 VAP may exhibit impaired ability to clear virus compared to those who do not, and that the lung 261 microbiome composition may be similarly impacted. 
Indeed, COVID-19 patients who developed VAP exhibited a significant reduction in

263 bacterial diversity of their airway microbiome up to three weeks before clinical signs of infection

264 (Shannon Diversity Index, p=0.012; Figure 6B). COVID-19 patients who developed VAP also had 265 lower airway microbiome compositions more closely resembling each other than those from 266 patients who did not develop VAP, across all timepoints since intubation (Bray Curtis index, $267 p=0.0033$; Figure 6C), suggesting community collapse precedes the development of VAP. All 268 patients received antibiotics prior to collection of the first sample, suggesting that antibiotic use 269 was not driving these differences (Table S1).

Discussion

Secondary bacterial pneumonia contributes to significant morbidity and mortality in 273 patients with primary viral lower respiratory tract infections ${ }^{1,3}$, but mechanisms governing 274 individual susceptibility to VAP have remained unclear. Few human cohort studies have evaluated 275 the immunologic underpinnings of VAP, and none have been reported in the context of COVID276 19, which is characterized by a dysregulated host response distinct from other viral 277 pneumonias ${ }^{14,18,19}$. To address this gap and probe mechanisms of VAP susceptibility in patients 278 with COVID-19, we carried out a systems biological assessment of host and microbial dynamics 279 of the lower respiratory tract.

280 Two days before VAP onset, a transcriptional signature consistent with bacterial infection 281 was observed. This finding suggests that host response changes can occur before clinical 282 recognition of pneumonia, highlighting the potential utility of the host transcriptome as a tool for 283 VAP surveillance. While intriguing, this observation did not provide an explanation for differential 284 susceptibility of some COVID-19 patients to post-viral pneumonia.

285 The discovery of an early suppressed antibacterial immune response in patients who later 286 developed VAP did however, offer a potential explanation. More than two weeks before VAP 287 onset, we observed a striking suppression of pathways related to both innate and adaptive 
288 immunity, including neutrophil degranulation, TLR signaling, complement activation, antigen 289 presentation, and T cell receptor and B receptor signaling, as well as cytokine signaling (e.g. IL290 1, IL-4, IL-12, IL-13 and IL-17). Comparison against uninfected, intubated controls confirmed the 291 previously described paradoxical impairment in immune signaling found in patients with severe 292 COVID-19 ${ }^{18}$, and suggested that VAP susceptibility may be the result of disproportionate 293 suppression of innate and adaptive pathways critical for antibacterial defense, resulting in 294 enhanced susceptibility to opportunistic secondary infections.

295 Animal models of influenza have provided insight into potential mechanisms of post-viral 296 pneumonia, although none have provided insight regarding why some individuals are more 297 susceptible than others. In mice inoculated with influenza, for instance, virus-induced type I IFN 298 suppresses neutrophil chemokines and impairs Th17 immunity, compromising effective clearance 299 of bacterial infections ${ }^{9,10}$. Interestingly, we also observed increased type I interferon signaling in 300 COVID-19 patients who weeks later developed VAP, and a strikingly similar impairment in Th17 301 signaling and other immune pathways. Desensitization to toll-like receptor (TLR) ligands after 302 influenza infection has also been documented ${ }^{20}$, which is congruent with the downregulation of 303 TLR signaling at the time of intubation observed in our bulk RNA-seq analyses.

$304 \quad$ Impaired bacterial clearance by alveolar macrophages was found to be driven by virus305 related IFN $\gamma$ production by $\mathrm{T}_{\text {cells }}{ }^{21}$ in a murine post-influenza model. In contrast, we found that 306 T cells from patients who later developed VAP expressed lower levels of IFN $\gamma$ at the time of 307 intubation. This difference may relate to species-specific variations in immune signaling or intrinsic 308 differences in the host response to influenza virus versus SARS-CoV-2 ${ }^{14,18}$. We asked whether certain cell types were responsible for driving the early suppression of 310 immune signaling observed in COVID-19 patients who went on to develop VAP. No significant 311 differences in proportions of the most abundant cell types - monocytes/macrophages, neutrophils 312 or T cells - was observed between patients with or without VAP at the time of intubation. This 
313 finding suggests that an impairment of immune cell recruitment was not causing these differences,

314 but rather significant gene expression differences within each of these immune cell populations.

315 In both the mono/mac and neutrophil populations, we observed broad downregulation of

316 the innate immune response, and initiation of the adaptive immune response, concordant with

317 global observations in bulk RNA-seq analyses. Further analysis revealed a downregulation of

318 monocyte to macrophage differentiation and neutrophil chemotaxis. Further, we noted a

319 downregulation of key pathways and transcription factors involved in antimicrobial immune

320 responses including iNOS in mono/macs, as well as NFKB and TREM1 in mono/macs and

321 neutrophils. Both bulk and scRNA-seq suggested an impairment in T cell recruitment, signaling,

322 and effector functions. Overall, our data suggest that while no difference in cell type populations

323 existed between groups, changes in the gene expression of mono/macs, neutrophils and T cells

324 contributes to immune suppression in COVID-19 patients who later develop VAP.

325 SARS-CoV-2 viral load correlates with interferon stimulated gene expression ${ }^{14,18}$ and thus

326 we initially hypothesized that differences in viral load between groups might relate to individual

327 VAP susceptibility. However, we found no difference between groups at the "early" timepoint.

328 Moreover, no differences existed in terms of immunosuppressive medication administration or

329 clinically diagnosed immunodeficiency, suggesting that other, still unidentified mechanisms

330 present at the time of intubation must underlie the marked suppression of immune gene

331 expression in COVID-19 patients who went on to develop VAP.

332 While no difference in viral load was observed at the time of intubation, the COVID-19

333 patients who developed VAP exhibited impaired viral clearance over the time-course of intubation.

334 This observation was corroborated by a prolonged antiviral type I interferon response at the "late"

335 timepoint (median of two days before VAP onset) in patients who developed VAP versus those

336 who did not, pointing to the persistence of suboptimal antiviral immunity in these patients. Early

337 induction of functional SARS-CoV-2 specific T cells is associated with faster viral clearance in 
338 COVID-19 patients ${ }^{22}$ and likewise, we observed impairments in T cell activation and signaling in 339 the VAP group, which further suggests a decreased ability to control the virus in these patients.

340 Respiratory viruses can reshape the human airway microbiome by modulating host 341 inflammatory responses ${ }^{23,24}$. In mouse models of influenza, the airway microbiome exhibits

342 expansion of several bacterial families during the course of viral infection as innate immunity is 343 suppressed $^{23}$. These changes increase the risk of secondary bacterial infection ${ }^{23}$ and have been 344 observed in patients with chronic obstructive pulmonary disease, where suppression of the innate 345 immune response in rhinovirus infected patients may be followed by bacterial superinfection ${ }^{25,26}$. 346 Similarly, the innate immune suppression observed in COVID-19 patients who developed

347 VAP was associated with airway microbiome collapse and the outgrowth of lung pathogens in 348 advance of clinical VAP diagnosis. This finding suggests that individual immune responses to 349 SARS-CoV-2 infection may drive a restructuring of the microbial community and increase 350 susceptibility to VAP (Figure 7). The resulting outgrowth of a VAP-associated bacterial pathogen 351 may elicit an antibacterial response, but the broader immunosuppressive state preceding this 352 response may be insufficient to control the development of clinical pneumonia. Those with a 353 lesser degree of immunosuppression may be able to respond faster and therefore control 354 opportunistic bacterial pathogens more effectively.

355 These findings may also have important implications for management of patients with 356 COVID-19 related acute respiratory failure, many of whom are now being treated with 357 corticosteroids plus/minus IL-6 receptor blocking agents. These agents may lead to further 358 suppression of the key pathways required for host response to secondary bacterial infection.

359 Thus, our results emphasize the need for ongoing vigilance for VAP in patients treated with potent 360 immunosuppressive agents, as well as the need to develop novel diagnostic and/or prognostic 361 approaches to identifying patients at highest risk. For instance, availability of molecular 362 biomarkers to assess a patient's risk of VAP at the time of intubation could reduce inappropriate 363 use of prophylactic antibiotics or immunomodulatory treatments, or signal a need for enhanced 
surveillance strategies. Signatures of immune dysfunction have been used as biomarkers to predict nosocomial infection in critically ill patients, ${ }^{27}$ although not in the context of viral infection.

Sample size is a limitation of this study; however, the reproducibility of our observations across both bulk and scRNA-seq analyses and the significant number of differentially expressed genes among the comparator groups support the validity of our conclusions. Because this study was limited to critically ill, intubated patients, we were unable to assess early stages of COVID19, which may provide additional insight regarding determinants of secondary bacterial infection.

371 Additionally, we were unable to assess whether epithelial cells contributed to VAP risk due to 372 enrichment for immune cells prior to scRNA-seq. With larger cohorts, the early detection of specific immune pathway suppression and microbiome collapse could be leveraged to develop

374 clinically useful models for identifying COVID-19 patients with increased susceptibility to secondary bacterial pneumonia.

\section{$377 \quad$ Materials and Methods}

\section{Study design, cohorts, enrollment and ethics approval}

We conducted a prospective case-control study of adults requiring mechanical ventilation

381 for COVID-19 with or without secondary bacterial pneumonia. We also evaluated control patients 382 requiring mechanical ventilation for other reasons who had no evidence of pulmonary infection 383 (Figure 1). Patients were enrolled in either of two prospective cohort studies of critically ill patients 384 at the University of California, San Francisco (UCSF) and Zuckerberg San Francisco General 385 Hospital between 07/2013 and 07/2020. Both cohort studies were approved by the UCSF 386 Institutional Review Board (IRB) under protocols 10-02701 (control patients, pre-COVID-19 387 pandemic) and 20-30497 (COVID-19 patients, COVID-19 Multiphenotyping for Effective Therapy 388 (COMET) study), respectively. Of the COVID-19 patients, 19 were co-enrolled in the National 
Institute of Allergy and Infectious Diseases-funded Immunophenotyping Assessment in a COVID19 Cohort (IMPACC) Network study.

For both the COVID-19 and control cohorts, if a patient met inclusion criteria, then a study coordinator or physician obtained written informed consent for enrollment from the patient or their surrogate. Patients or their surrogates were provided with detailed written and verbal information about the goals of the study, the data and specimens that would be collected, and the potential risks to the subject. Patients and their surrogates were also informed that there would be no benefit to them from being enrolled in the study and that they may withdraw informed consent at any time during the course of the study. All questions were answered, and informed consent documented by obtaining the signature of the patient or their surrogate on the consent document (or during the COVID-19 pandemic, the IRB-approved electronic equivalent, to enable touchless consent).

Many critically ill patients are unconscious at the time of intensive care unit (ICU) admission due to their underlying illness and/or are endotracheally intubated for airway management or acute respiratory failure. The patients who are not unconscious are often in pain and may have acute delirium due to critical illness and/or medications. For these reasons, many subjects are unable to provide informed consent at the time of enrollment. Because this study could not practically be done otherwise and was deemed to be minimal risk by the UCSF IRB, if 407 a patient was unable and a surrogate was not available to provide consent, patients were enrolled 408 with waiver of initial consent, including the collection of biological samples.

410 enrollment, our study team was permitted to collect biological samples as well as clinical data

411 from the medical record obtained prior to consent. Surrogate consent was vigorously pursued for 412 all patients; moreover, each patient was regularly examined to determine if and when s/he was 413 able to consent for him/herself, and the nursing and ICU staff were contacted daily for information 
414 about surrogates' availability. For patients whose surrogates provided informed consent, follow-

415 up consent was subsequently obtained from the patient if they survived their acute illness and

416 regained the ability to consent. For subjects who died prior to the consent being obtained, a full

417 waiver of consent was approved by the UCSF IRB for both cohort studies. Lack of a surrogate to

418 provide consent is common in critically ill patients. To address this, the UCSF IRB also approved

419 a full waiver of consent for subjects in the COVID-19 cohort who remained unable to provide

420 informed consent and had no contactable surrogate identified within 28 days. Before utilizing this

421 waiver, we made and documented at least three separate attempts to identify and contact the

422 patient or surrogate over a month-long period. While most patients enrolled were consented by

423 typical processes, three died prior to consent being obtained, and five were included with a full

424 waiver of consent due to lack of ability to consent and lack of contactable surrogate. No personally

425 identifiable information has been included as part of this manuscript for any enrolled patients.

\section{Ventilator-associated pneumonia adjudication}

A total of 84 adults who required intubation for severe COVID-19 (Cohort 1) and who had

429 available TA samples were considered for inclusion in the study (Figure 1). Patients who met the 430 Centers for Disease Control (CDC) definition for VAP ${ }^{13}$ with a positive bacterial sputum culture 431 were adjudicated as having VAP for the purpose of the study $(\mathrm{N}=16)$; patients who did not meet 432 these criteria, and for whom there was no sustained clinical suspicion for bacterial pneumonia 433 during the admission, were categorized as No-VAP $(\mathrm{N}=17)$. VAP and No-VAP patients for whom 434 samples at the time-points of interest were available were included in the primary analyses (VAP: $435 \mathrm{~N}=10$; No-VAP: $\mathrm{N}=13$ ). Patients who met CDC-VAP criteria but had negative TA cultures were 436 included in a secondary supplementary analysis only $(\mathrm{N}=5)$. All other patients were excluded, 437 including patients with clinically-suspected bacterial pneumonia who did not meet CDC VAP 438 criteria. Eight intubated patients from a recent study ${ }^{18}$ (Cohort 2) were included as controls and 
were selected because they had previously been adjudicated as having no evidence of lower

440 respiratory tract infection. This group included four patients with the acute respiratory distress

441 syndrome (ARDS) due to non-infectious etiologies, and four patients without ARDS who were

442 intubated for other reasons (subdural hematoma $(N=1)$, retroperitoneal hemorrhage $(N=1)$, or

443 neurosurgical procedures $(\mathrm{N}=2))$.

444

\section{Tracheal aspirate sampling}

446 Following enrollment, tracheal aspirate (TA) was collected (periodically following 447 intubation for Study 1, or once within 3 days of intubation for Study 2), without addition of saline 448 wash, and either a) mixed 1:1 with DNA/RNA shield (Zymo Research) for bulk RNA-seq or b) 449 immediately processed in a biosafety level 3 laboratory (BSL3) for scRNA-seq analysis.

\section{Bulk RNA sequencing and host transcriptome analysis}

RNA sequencing

To evaluate host and microbial gene expression, metatranscriptomic next generation RNA sequencing (RNA-seq) was performed on TA specimens. Following RNA extraction (Zymo

456 Pathogen Magbead Kit) and DNase treatment, human cytosolic and mitochondrial ribosomal RNA 457 was depleted using FastSelect (Qiagen). To control for background contamination, we included 458 negative controls (water and HeLa cell RNA) as well as positive controls (spike-in RNA standards 459 from the External RNA Controls Consortium (ERCC)) $)^{28}$. RNA was then fragmented and 460 underwent library preparation using the NEBNext Ultra II RNA-seq Kit (New England BioLabs).

461 Libraries underwent 146 nucleotide paired-end Illumina sequencing on an Illumina Novaseq 6000. 462 
Following demultiplexing, sequencing reads were pseudo-aligned with kallisto ${ }^{29}$ to an index consisting of all transcripts associated with human protein coding genes (ENSEMBL v.

466 99), cytosolic and mitochondrial ribosomal RNA sequences and the sequences of ERCC RNA 467 standards. Gene-level counts were generated from the transcript-level abundance estimates 468 using the R package tximport ${ }^{30}$, with the scaledTPM method. Samples retained in the dataset 469 had a total of at least 1,000,000 estimated counts associated with transcripts of protein coding 470 genes.

471 Genes were retained for differential expression analysis if they had counts in at least $30 \%$ 472 of samples. Differential expression analysis was performed using the R package DESeq2 ${ }^{31}$. We 473 modeled the expression of individual genes using the design formula VAPgroup, where VAP 474 groups were "VAP-early", "No VAP-early", "VAP-late" and "No VAP-late" and used the results() 475 function to extract a specific contrast. Separate comparisons to the control group were performed 476 using the design formula COVID-19-status to compare positive and negative patients.

477 Significant genes were identified using a Benjamini-Hochberg false discovery rate (FDR)

$478<0.1$. We generated heatmaps of the top 50 differentially expressed genes by FDR. For 479 visualization, gene expression was normalized using the regularized log transformation, centered, 480 and scaled prior to clustering. Heatmaps were generated using the pheatmap package. Columns 481 were clustered using Euclidean distance and rows were clustered using Pearson correlation. 482 Differential expression analysis results are provided in (Supplementary data file 1).

\section{$484 \quad$ Pathway analysis}

485 Gene set enrichment analyses (GSEA) were performed using the fgseaMultilevel function 486 in the R package fgsea ${ }^{32}$ and REACTOME pathways ${ }^{33}$ with a minimum size of 10 genes and a 487 maximum size of 1,500 genes. All genes were included in the comparison, pre-ranked by the test 488 statistic. Significant pathways were defined as those with a Benjamini-Hochberg adjusted p-value 
$<0.05$. Ingenuity Pathway Analysis (IPA) Canonical Pathway and Upstream Regulator Analysis ${ }^{34}$

490 was employed on genes with $p<0.1$ and ranked by the test statistic to identify cytokine regulators.

491 Significant IPA results were defined as those with a Z-score absolute value greater than 2 and an

492 overlap $P$ value $<0.05$. The gene sets in figures were selected to reduce redundancy and highlight

493 diverse biological functions. Full GSEA and IPA results are provided in (Supplementary data

494 files 2 and 3 ).

495 Longitudinal pathway analysis was performed using all available TA samples spanning 496 post-intubation to VAP onset for all patients included in the bulk RNA-seq analysis. Analysis was 497 restricted to samples with at least $1,000,000$ human protein coding transcripts. Pathways of 498 interest were selected from the significant GSEA results of the comparison of VAP vs. No-VAP 499 patients in the "early" time-point. The top 20 leading edge genes were selected from each pathway 500 for analysis. To calculate a Z-score for each gene, expression was normalized using the variance 501 stabilizing transformation (VST), centered, and scaled. A pathway Z-score was calculated by 502 averaging the 20 gene Z-scores. Multiple Z-scores per patient at a given time interval were 503 averaged so that each patient corresponds to one datapoint at each interval. Statistical 504 significance of pathway expression over time between VAP and No-VAP groups was calculated 505 using a two-way analysis of variance (ANOVA) in GraphPad PRISM.

\section{Single cell RNA sequencing and transcriptome analysis}

511 temperature, followed by passage through a $70 \mu \mathrm{M}$ filter. Cells were pelleted at $350 \mathrm{~g} 4 \mathrm{C}$ for 10 512 minutes, resuspended in PBS with $2 \mathrm{mM}$ EDTA and $0.5 \% \mathrm{BSA}$, and manually counted on a 513 hemocytometer. Cells were stained with MojoSort Human CD45 and purified by the 514 manufacturer's protocol (Biolegend). After CD45 positive selection, cells were manually counted 
515 with trypan blue on a hemocytometer. Using a $V(D) J$ v1.1 kit according to the manufacturer's

516 protocol, samples were loaded on a 10X Genomics Chip A without multiplexing, aiming to capture

51710,000 cells (10X Genomics). Libraries underwent paired end 150 base pair sequencing on an

518 Illumina NovaSeq6000.

519 Raw sequencing reads were aligned to GRCh38 using the STAR aligner ${ }^{35}$. Cell barcodes 520 were then determined based upon UMI count distribution. Read count matrices were generated 521 through the 10X genomics cellranger pipeline v3.0. Data was processed and analyzed using the 522 Scanpy $v 1 \cdot 6^{36}$. Cells that had $<200$ genes and had greater than 30,000 counts were filtered.

523 Mitochondrial genes were removed and multi-sample integration was performed using Harmony $524 \mathrm{v} 0.1 \cdot 4^{37}$. Differential expression was performed using MAST v1.16.0 $0^{38}$. Due to the significantly greater number of differentially expressed genes in scRNA-seq analyses, we used a more restrictive cutoff of FDR $<0.05$ for significant genes. Differential expression analysis results are detailed in (Supplementary data file 4).

Pathway analysis

Ingenuity Pathway Analysis (IPA) Canonical Pathway and Upstream Regulator Analysis ${ }^{34}$

531 was employed on genes with $p<0.05$ and ranked by log2foldchange to identify canonical pathways

532 and cytokine regulators. We utilized a more restrictive $p$ value cutoff for scRNA-seq to ensure a

533 similar number of genes were input into IPA. Significant IPA results were defined as those with a

534 Z-score absolute value greater than 2 and an overlap $P$ value $<0.05$. The gene sets in figures

535 were selected to reduce redundancy and highlight diverse biological functions. Full GSEA and 536 IPA results are provided in (Supplementary data files 5 and 6).

\section{Lung microbiome analysis}

539 RNA from tracheal aspirates was sequenced as described above. Taxonomic alignments 540 were obtained from raw sequencing reads using the IDseq pipeline ${ }^{39,40}$, which performs quality 
541 filtration and removal of human reads followed by reference-based taxonomic alignment at both

542 the nucleotide and amino acid level against sequences in the National Center for Biotechnology

543 Information (NCBI) nucleotide (NT) and non-redundant (NR) databases, followed by assembly of

544 reads matching each taxon detected. Taxonomic alignments underwent background correction

545 for environmental contaminants (see below), viruses were excluded, and data was then

546 aggregated to the genus level before calculating diversity metrics. Alpha diversity (Shannon's

547 Diversity Index) and beta diversity (Bray-Curtis dissimilarity) were calculated and the latter plotted

548 using non-metric multidimensional scaling (NDMS). Comparison of alpha and beta diversity over

549 time between VAP and No-VAP groups was calculated using a two-way analysis of variance

550 (ANOVA) in GraphPad PRISM.

$\underline{\text { Identification and mitigation of environmental contaminants }}$

To minimize inaccurate taxonomic assignments due to environmental and reagent derived

554 contaminants, non-templated "water only" and HeLa cell RNA controls were processed with each

555 group of samples that underwent nucleic acid extraction. These were included, as well as positive

556 control clinical samples, with each sequencing run. Negative control samples enabled estimation

557 of the number of background reads expected for each taxon. A previously developed negative

558 binomial model ${ }^{14}$ was employed to identify taxa with NT sequencing alignments present at an

559 abundance significantly greater compared to negative water controls. This was done by modeling

560 the number of background reads as a negative binomial distribution, with mean and dispersion

561 fitted on the negative controls. For each batch (sequencing run) and taxon, we estimated the

562 mean parameter of the negative binomial by averaging the read counts across all negative

563 controls, slightly regularizing this estimate by including the global average (across all batches) as

564 an additional sample. We estimated a single dispersion parameter across all taxa and batches, 565 using the functions glm.nb() and theta.md() from the R package MASS ${ }^{41}$. Taxa that achieved a p566 value $<0.01$ were carried forward 


\section{References}

568 1. Falsey, A. R. et al. Bacterial Complications of Respiratory Tract Viral Illness: A

569 Comprehensive Evaluation. The Journal of Infectious Diseases 208, 432-441 (2013).

570 2. Morens, D. M., Taubenberger, J. K. \& Fauci, A. S. Predominant role of bacterial pneumonia

571 as a cause of death in pandemic influenza: implications for pandemic influenza

572 preparedness. J Infect Dis 198, 962-970 (2008).

573 3. Rouzé, A. et al. Relationship between SARS-CoV-2 infection and the incidence of ventilator-

574 associated lower respiratory tract infections: a European multicenter cohort study. Intensive

$575 \quad$ Care Med 1-11 (2021) doi:10.1007/s00134-020-06323-9.

576 4. Luyt, C.-E. et al. Ventilator-associated pneumonia in patients with SARS-CoV-2-associated

577 acute respiratory distress syndrome requiring ECMO: a retrospective cohort study. Ann

$578 \quad$ Intensive Care 10, 158 (2020).

579 5. Bardi, T. et al. Nosocomial infections associated to COVID-19 in the intensive care unit:

$580 \quad$ clinical characteristics and outcome. Eur J Clin Microbiol Infect Dis (2021)

581 doi:10.1007/s10096-020-04142-w.

582 6. Maes, M. et al. Ventilator-associated pneumonia in critically ill patients with COVID-19. Crit

$583 \quad$ Care 25, 25 (2021).

584 7. Søgaard, K. K. et al. Community-acquired and hospital-acquired respiratory tract infection

585 and bloodstream infection in patients hospitalized with COVID-19 pneumonia. J Intensive

$586 \quad$ Care $\mathbf{9},(2021)$.

587 8. Metzger, D. W. \& Sun, K. Immune Dysfunction and Bacterial Co-Infections following

588 Influenza. J Immunol 191, 2047-2052 (2013).

589 9. Shahangian, A. et al. Type I IFNs mediate development of postinfluenza bacterial pneumonia

$590 \quad$ in mice. J Clin Invest 119, 1910-1920 (2009).

591 10. Kudva, A. et al. Influenza A inhibits Th17-mediated host defense against bacterial

592 pneumonia in mice. J Immunol 186, 1666-1674 (2011). 
593 11. Langelier, C. et al. Integrating host response and unbiased microbe detection for lower 594 respiratory tract infection diagnosis in critically ill adults. Proc Natl Acad Sci U S A 115, $595 \quad$ E12353-E12362 (2018).

596 12. Flanagan, J. L. et al. Loss of bacterial diversity during antibiotic treatment of intubated 597 patients colonized with Pseudomonas aeruginosa. J Clin Microbiol 45, 1954-1962 (2007).

598 13. CDC-NHSN. Pneumonia (Ventilator-associated [VAP] and non-ventilator-associated 599 Pneumonia [PNEU]) Event.

600 14. Mick, E. et al. Upper airway gene expression reveals suppressed immune responses to 601 SARS-CoV-2 compared with other respiratory viruses. Nature Communications 11, 5854 $602 \quad(2020)$

603 15. Wein, A. N. et al. CXCR6 regulates localization of tissue-resident memory CD8 T cells to the 604 airways. Journal of Experimental Medicine 216, 2748-2762 (2019).

605 16. Grau, M. et al. Antigen-Induced but Not Innate Memory CD8 T Cells Express NKG2D and 606 Are Recruited to the Lung Parenchyma upon Viral Infection. The Journal of Immunology $607 \quad 200,3635-3646(2018)$.

608 17. Smith-Garvin, J. E., Koretzky, G. A. \& Jordan, M. S. T cell activation. Annu Rev Immunol 27, $609 \quad 591-619(2009)$.

610 18. Sarma, A. et al. COVID-19 ARDS is characterized by a dysregulated host response that 611 differs from cytokine storm and is modified by dexamethasone. Res Sq (2021) 612 doi:10.21203/rs.3.rs-141578/v1.

613 19. Blanco-Melo, Daniel. Imbalanced host response to SARS-CoV-2 drives development of 614 COVID-19. Cell. doi:10.1016/j.cell.2020.04.026.

615 20. Didierlaurent, A. et al. Sustained desensitization to bacterial Toll-like receptor ligands after 616 resolution of respiratory influenza infection. J Exp Med 205, 323-329 (2008).

617 21. Sun, K. \& Metzger, D. W. Inhibition of pulmonary antibacterial defense by interferon-gamma 618 during recovery from influenza infection. Nat Med 14, 558-564 (2008). 
22. Tan, A. T. et al. Early induction of functional SARS-CoV-2-specific T cells associates with rapid viral clearance and mild disease in COVID-19 patients. Cell Reports 34, 108728 (2021).

23. Goulding, J. et al. Lowering the Threshold of Lung Innate Immune Cell Activation Alters Susceptibility to Secondary Bacterial Superinfection. J Infect Dis 204, 1086-1094 (2011).

24. Man, W. H., de Steenhuijsen Piters, W. A. A. \& Bogaert, D. The microbiota of the respiratory

26. Molyneaux, P. L. et al. Outgrowth of the Bacterial Airway Microbiome after Rhinovirus Exacerbation of Chronic Obstructive Pulmonary Disease. Am J Respir Crit Care Med 188,

27. Conway Morris, A. et al. Cell-surface signatures of immune dysfunction risk-stratify critically ill patients: INFECT study. Intensive Care Med 44, 627-635 (2018).

28. Pine, P. S. et al. Evaluation of the External RNA Controls Consortium (ERCC) reference material using a modified Latin square design. BMC Biotechnol 16, 54 (2016).

29. Bray, N. L., Pimentel, H., Melsted, P. \& Pachter, L. Near-optimal probabilistic RNA-seq quantification. Nature Biotechnology 34, 525-527 (2016).

30. Soneson, C., Love, M. I. \& Robinson, M. D. Differential analyses for RNA-seq: transcriptlevel estimates improve gene-level inferences. F1000Res 4, 1521 (2015).

31. Love, M. I., Huber, W. \& Anders, S. Moderated estimation of fold change and dispersion for RNA-seq data with DESeq2. Genome Biol 15, 550 (2014).

32. Korotkevich, G., Sukhov, V. \& Sergushichev, A. Fast gene set enrichment analysis. bioRxiv 060012 (2019) doi:10.1101/060012. 
644 33. Croft, D. et al. The Reactome pathway knowledgebase. Nucleic Acids Res 42, D472-477 $645 \quad$ (2014).

646 34. Krämer, A., Green, J., Pollard, J. \& Tugendreich, S. Causal analysis approaches in 647 Ingenuity Pathway Analysis. Bioinformatics 30, 523-530 (2014).

648 35. Dobin, A. et al. STAR: ultrafast universal RNA-seq aligner. Bioinformatics 29, 15-21 (2013).

649 36. Wolf, F. A., Angerer, P. \& Theis, F. J. SCANPY : large-scale single-cell gene expression 650 data analysis. Genome Biology 19, 15 (2018).

651 37. Korsunsky, I. et al. Fast, sensitive, and accurate integration of single cell data with 652 Harmony. bioRxiv 461954 (2018) doi:10.1101/461954.

653 38. Finak, G. et al. MAST: a flexible statistical framework for assessing transcriptional changes

654 and characterizing heterogeneity in single-cell RNA sequencing data. Genome Biol 16, 278 655 (2015).

656 39. Ramesh, A. et al. Metagenomic next-generation sequencing of samples from pediatric 657 febrile illness in Tororo, Uganda. PLoS One 14, e0218318 (2019).

658 40. Kalantar, K. L. et al. IDseq-An open source cloud-based pipeline and analysis service for 659 metagenomic pathogen detection and monitoring. Gigascience 9, (2020).

660 41. Venables, W. N. \& Ripley, B. D. Modern Applied Statistics with S. (Springer-Verlag, 2002). 661 doi:10.1007/978-0-387-21706-2.

662

663

664

665 
Acknowledgements: This study was performed with support from the National Institute of Allergy

667 and Infectious Diseases-sponsored Immunophenotyping Assessment in a COVID-19 Cohort

668 (IMPACC) Network. We gratefully appreciate support from Amy Kistler, PhD, Jack Kamm, PhD,

669 Angela Deitweiller, PhD, Saharai Caldera, BS and Maira Phelps BS.

670

671 Funding: Funding for the COMET cohort enrollment, sample collection and data analysis derived 672 from: K23HL138461-01A1 (CL), K24HL137013 (PGW), F32 HL151117 (AS), R35 HL140026

673 (CSC), NIAID U19AI077439 (DJE), Chan Zuckerberg Biohub (AB, JLD). The UCSF IMPACC site 674 was funded by NIAID U19AI077439 (DJE). Funding for enrollment of COMET participants not 675 enrolled in IMPACC and for all sample collection and data analysis derived from K23HL138461676 01A1 (CL), K24HL137013 (PGW), F32 HL151117 (AS), R35 HL140026 (CSC), and the Chan 677 Zuckerberg Biohub (AB, JLD). Philanthropic support was provided from Mark and Carrie Casey, 678 Julia and Kevin Hartz, Carl Kawaja and Wendy Holcombe, Eric Keisman and Linda Nevin, Martin 679 and Leesa Romo, Three Sisters Foundation, Diana Wagner and Jerry Yang and Akiko Yamazaki.

681 Author contributions:

682 Conceptualization: CRL, AT, BSZ, AB, CD, SL, ER, OR, CSC

683 Methodology: CRL, AT, BSZ, AB, CD, SL, ER, OR

684 Data acquisition: RG, AJ, PHS, TJD, BSZ, AB, NJ

685 Formal analysis: AT, BSZ, AB, CD, SL, ER, EM

686 Investigation: BSZ, AB, CD, SS, CSC, DJE

687 Funding acquisition: CRL, CSC, JLD, DJE

688 Supervision: CRL, OR, NN, JLD, CSC, DJE

689 Writing - original draft: AT, BSZ, AB, CD, SL, ER, CRL

690 Writing - review \& editing: All authors

691 
692 Competing interests: Authors declare that they have no competing interests.

693

694 Materials and Correspondence: Correspondence and material requests should be addressed to 695 Chaz Langelier (chaz.langelier@ucsf.edu).

696

697 Data and materials availability: Host gene expression data are available under NCBI GEO 698 accession number GSE168019 for bulk RNA-seq and GSE168018 for scRNA-seq. Raw microbial 699 sequencing alignments are available from NCBI SRA under BioProject PRJNA704082. Code 700 used for differential expression analysis is available at https://github.com/bspeco/VAPinCOVID19 


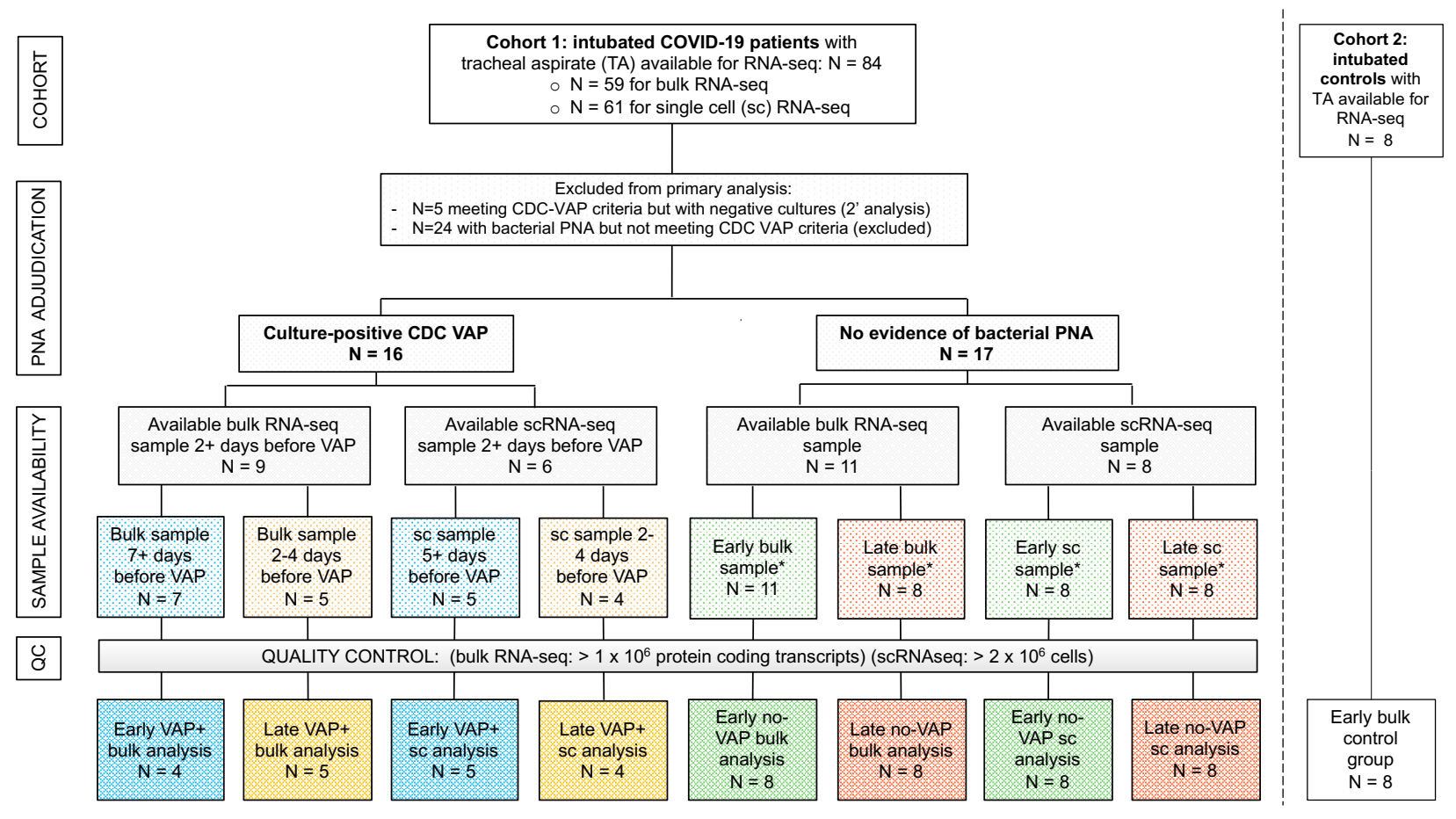

Figure 1: Study flowchart.

Two patient cohorts were studied. Cohort 1 consisted of COVID-19 patients from the COVID Multiphenotyping for Effective Therapies (COMET) / Immunophenotyping Assessment in a COVID-19 Cohort (IMPACC) studies (described in Methods). Cohort 2 consisted of critically ill intubated control patients from a prior prospective cohort study led by our research group ${ }^{18}$. The "early" samples were the first available tracheal aspirate specimens after intubation. For COVID19 patients who developed VAP, the "late" samples were obtained a median of two days before VAP onset. Timing of sample collection with respect to VAP versus No-VAP groups was matched at "early" and "late" time points. Controls included eight critically ill, mechanically ventilated patients without LRTI. All COVID-19 patients included in the primary bulk analysis were also included in the longitudinal host expression and microbiome analyses. Abbreviations: $\mathrm{VAP}=$ ventilator-associated pneumonia; $\mathrm{TA}=$ tracheal aspirate; $\mathrm{QC}=$ quality control; sc or scRNAseq= single cell RNA sequencing; $P N A=$ pneumonia; $C D C=$ United States Centers for Disease Control and Prevention. 
Figure 2
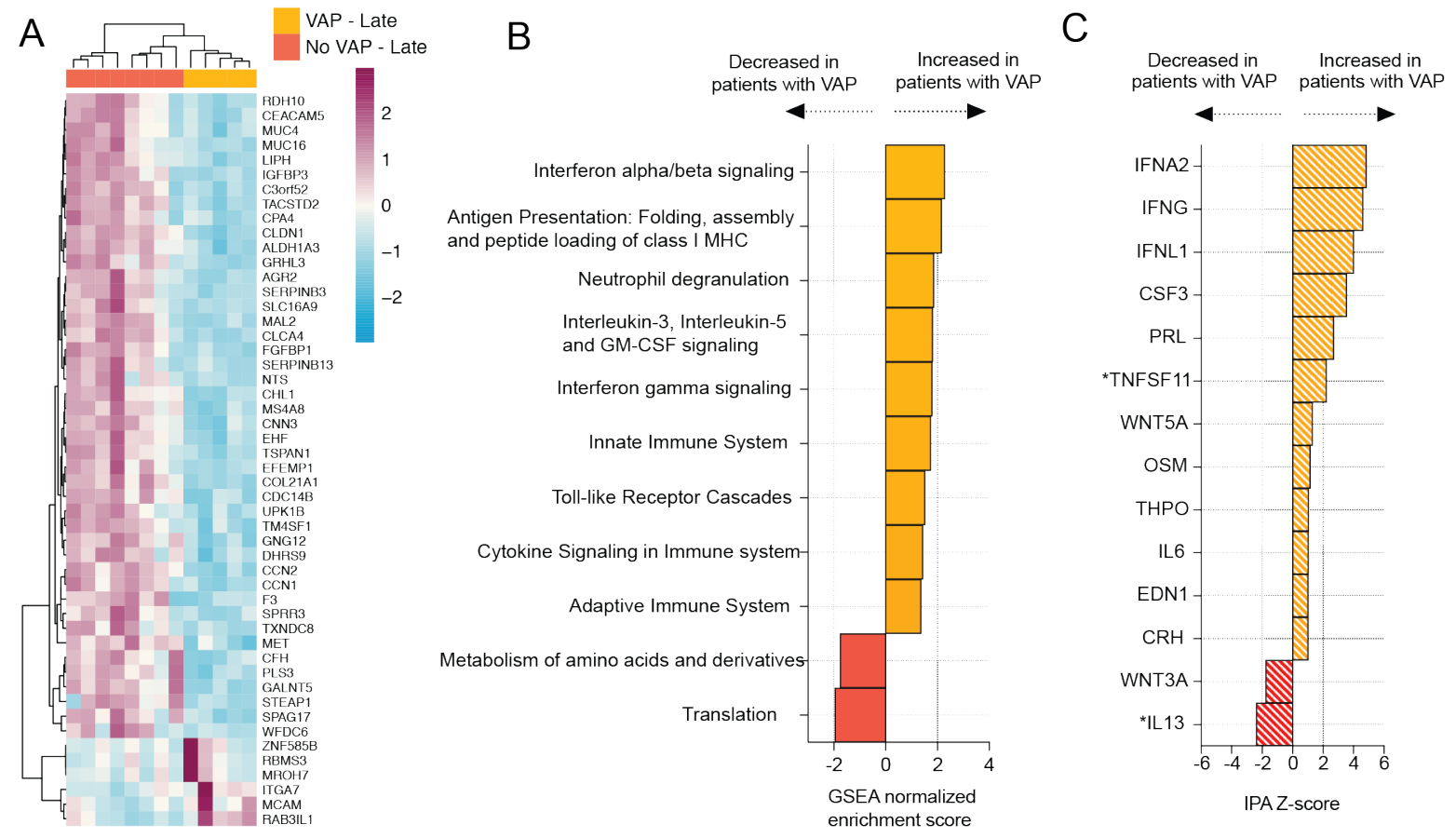

Figure 2: COVID-19 VAP is associated with a lower respiratory tract transcriptional signature of bacterial infection 2 days before VAP onset.

A) Heatmap of the top 50 differentially expressed genes by adjusted P-value between COVID-19 patients who developed VAP (yellow) versus those who did not (red) at the "late" time-point, 2 days before the onset of VAP, from bulk RNA-seq. B) Gene set enrichment analysis (GSEA) at the "late" time-point based on differential gene expression analyses. GSEA results were considered significant with an adjusted P-value $<0.05$. C) Ingenuity Pathway Analysis (IPA) of upstream cytokines at the "late" time-point based on differential gene expression analyses. IPA results were considered significant with a Z-score absolute value $>2$ and overlap P-value $<0.05$. ${ }^{*}$ Denotes cytokines with an overlap P-value $<0.1$. All pathways and cytokines are shown in Supplementary data files 2 and 3 . 


\section{Figure 3}
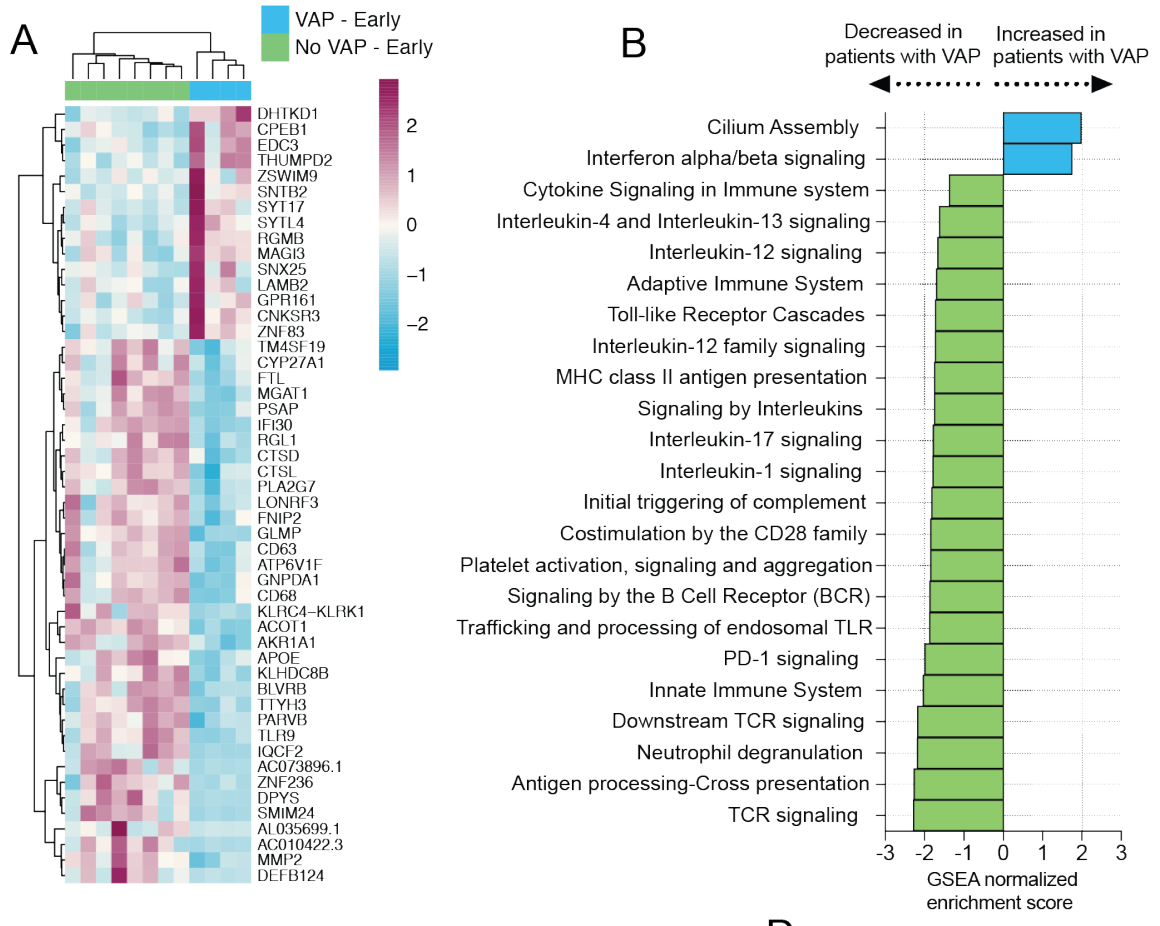

C

$$
\begin{aligned}
& \text { VAP } \\
& \text { No VAP }
\end{aligned}
$$

Decreased Increased
$4 \ldots \ldots \ldots \ldots \ldots \ldots$

vs. Uninfected controls

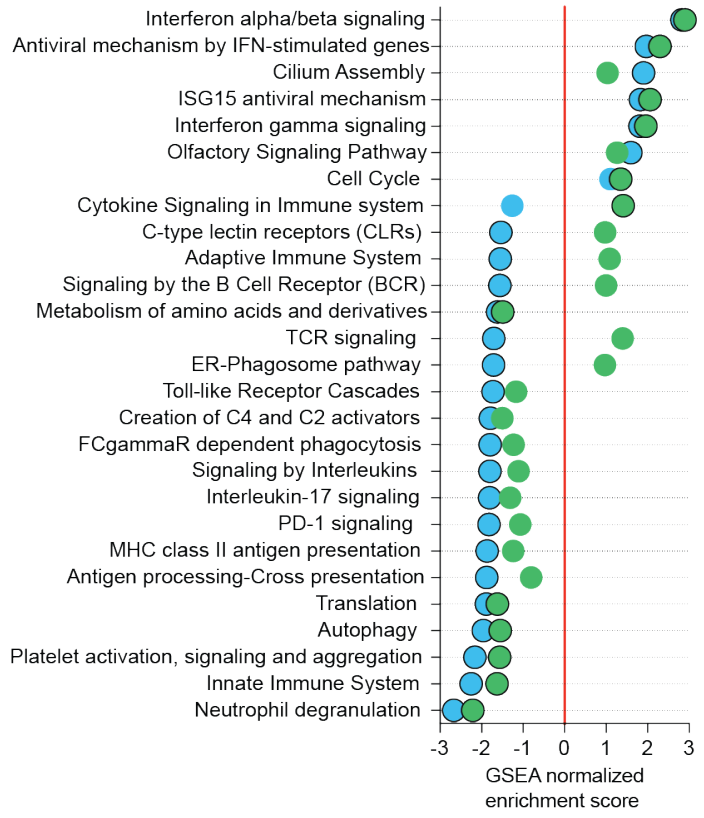
Decreased in
patients with VAP patients with VAP
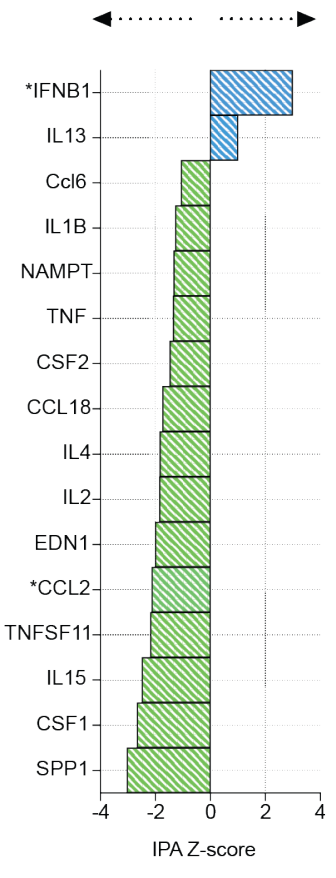
Figure 3: COVID-19 patients who develop VAP have attenuated immune signaling in the lower respiratory tract two weeks before onset of secondary bacterial pneumonia.

A) Heatmap of the top 50 differentially expressed genes by adjusted P-value between COVID-19 patients who developed VAP (blue) versus those who did not (green) at the "early" time-point from bulk RNA-seq. B) Gene set enrichment analysis at the "early" time-point based on differential gene expression analyses. GSEA results were considered significant with an adjusted P-value $<0.05$. C) Expression of GSEA pathways at the "early" time-point with respect to a baseline of uninfected, intubated controls. Pathways were selected from the GSEA results if they had an adjusted P-value $<0.05$ in at least one of the comparisons (VAP vs controls or No-VAP vs controls). Pathways with an adjusted P-value $<0.05$ when compared to controls are indicated by circles with a black outline. D) Ingenuity Pathway Analysis (IPA) of upstream cytokines at the "early" time-point based on differential gene expression analyses. IPA results were considered significant with a Z-score absolute value $>2$ and overlap P-value $<0.05$. *Denotes cytokines with an overlap P-value $<0.1$. All pathways and cytokines are shown in Supplementary data files 2 and 3. 
Figure 4

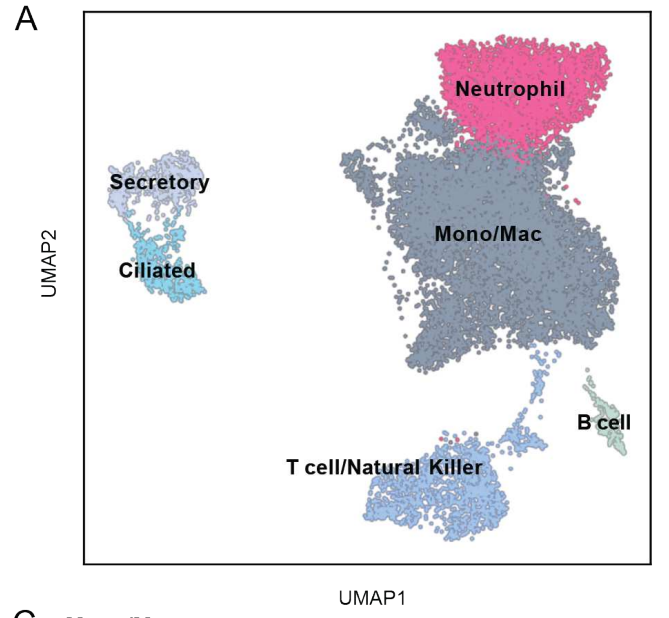

\section{Mono/Mac}

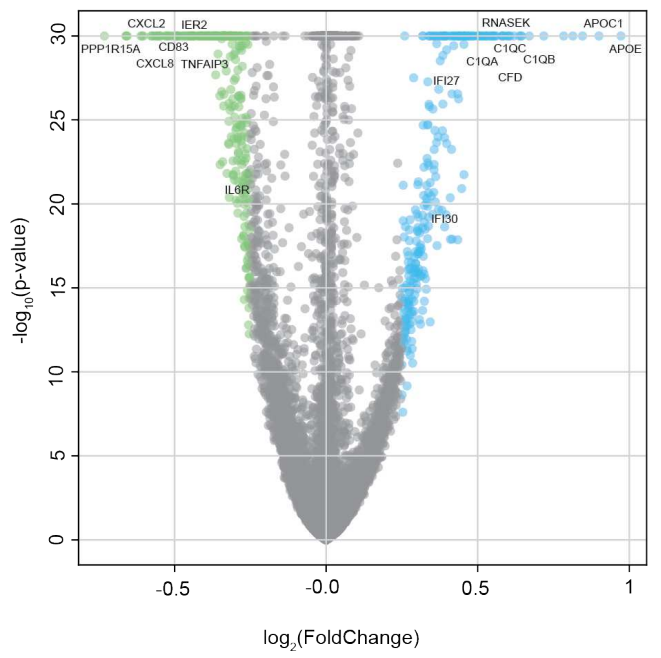

F Neutrophils

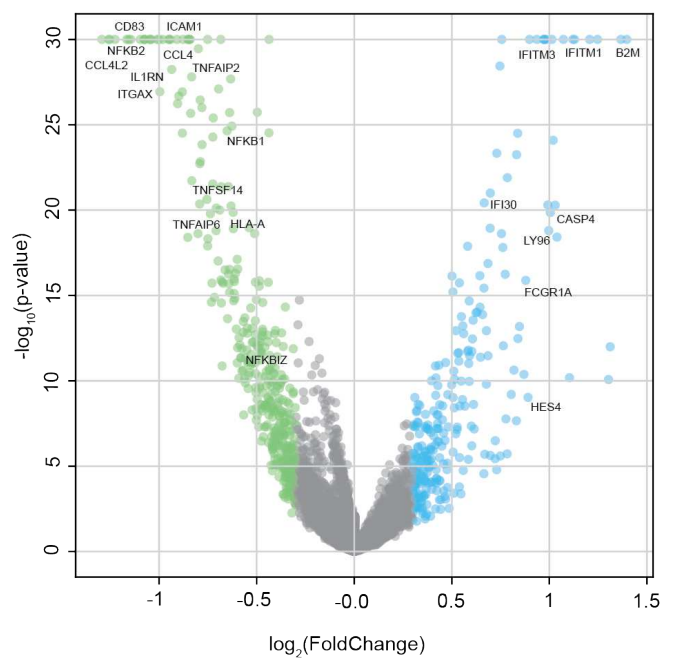

B

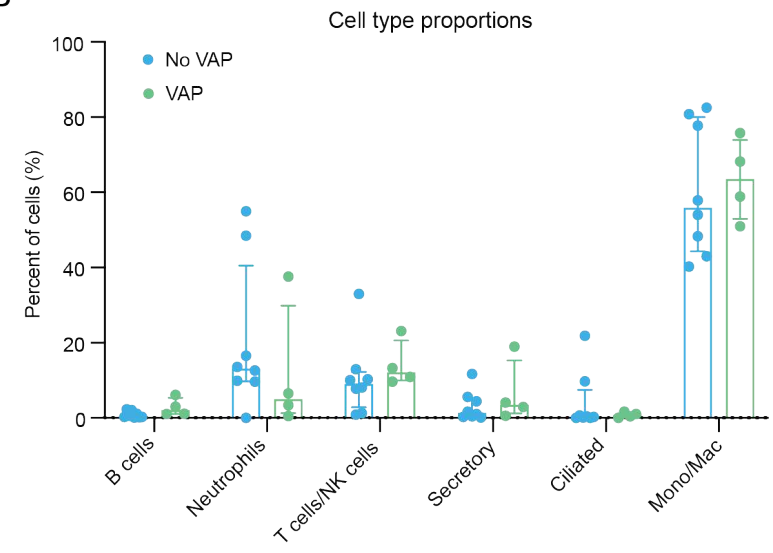

D

$E$

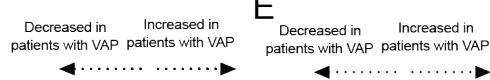

Oxidative Phosphorylation

$$
\text { Glutathio }
$$$$
\begin{array}{r}
\text { LXR/RXR Activation- } \\
\text { Glutathione-mediated Detoxification- }
\end{array}
$$$$
\text { Glycolysis I- }
$$

Role of IL-17F in Allergic Infl. Airway Diseases -

B Cell Activating Factor Signaling-

Toll-like Receptor Signaling -

JAK/Stat Signaling-

p38 MAPK Signaling-

Th17 Activation Pathway

TNFR2 Signaling-

NF-kB Activation by Viruses -

LPS/L-1 Mediated Inhibition of RXR Function -

HMGB1 Signaling-

IL-1 Signaling -

IL-15 Production-

iNOS Signaling -

Acute Phase Response Signaling TREM1 Signaling-

$$
\text { IL-6 Signaling- }
$$

\section{G}

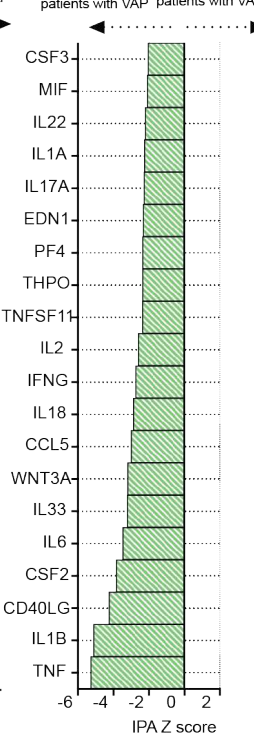

$\mathrm{H}$
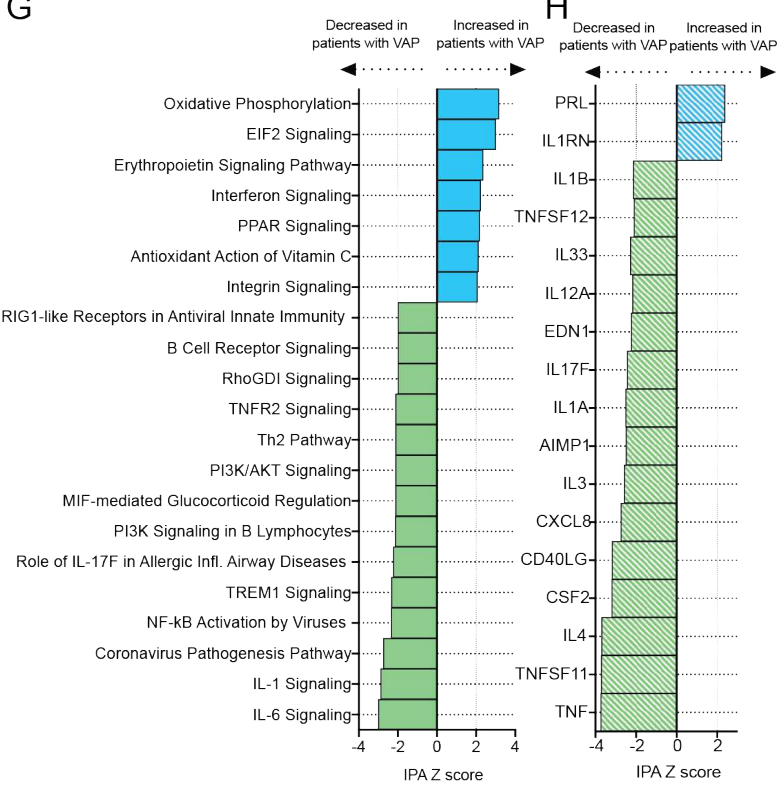
Figure 4: scRNA-seq demonstrates that COVID-19 VAP is associated with early impaired anti-bacterial immune signaling in lower respiratory tract monocytes, macrophages and neutrophils.

A) UMAP of single cell RNA-seq data from patients that do or do not develop VAP at the "early" time-point, annotated by cell type. B) Cell type proportions in single cell RNA-seq from VAP and No-VAP patients at the "early" time-point. Bars represent the median with IQR. Statistical significance was determined by Mann-Whitney tests. None of the cell types were significantly different with a $p$-value $<0.05$. The p-values for each cell type are as follows: B cells: 0.073 ; Neutrophils: 0.28; T/NK cells: 0.21; Secretory: 0.46; Ciliated: 0.94, and Mono/Mac: 0.81. C) Volcano plot displaying the differentially expressed genes between VAP and No-VAP patients in monocytes and macrophages. D) Ingenuity Pathway Analysis (IPA) of key canonical pathways and upstream cytokines based on differential gene expression analysis in monocytes and macrophages of patients who develop VAP versus those who do not, with adjusted $p$-values < 0.05 . Only significant pathways (IPA Z-score of $>2$ or $<-2$ and overlap p-value $<0.05$ ) are shown. E) Volcano plot displaying the differentially expressed genes between VAP and No-VAP patients in neutrophils. F) IPA of canonical pathways and upstream cytokines based on differential gene expression analysis in neutrophils of patients who develop VAP versus those who do not, with adjusted $p$-values $<0.05$. Only significant pathways (IPA Z-score of $>2$ or $<-2$ and overlap $p$-value $<0.05)$ are shown. All pathways and cytokines are shown in Supplementary data files 5 and 6. 
Figure 5
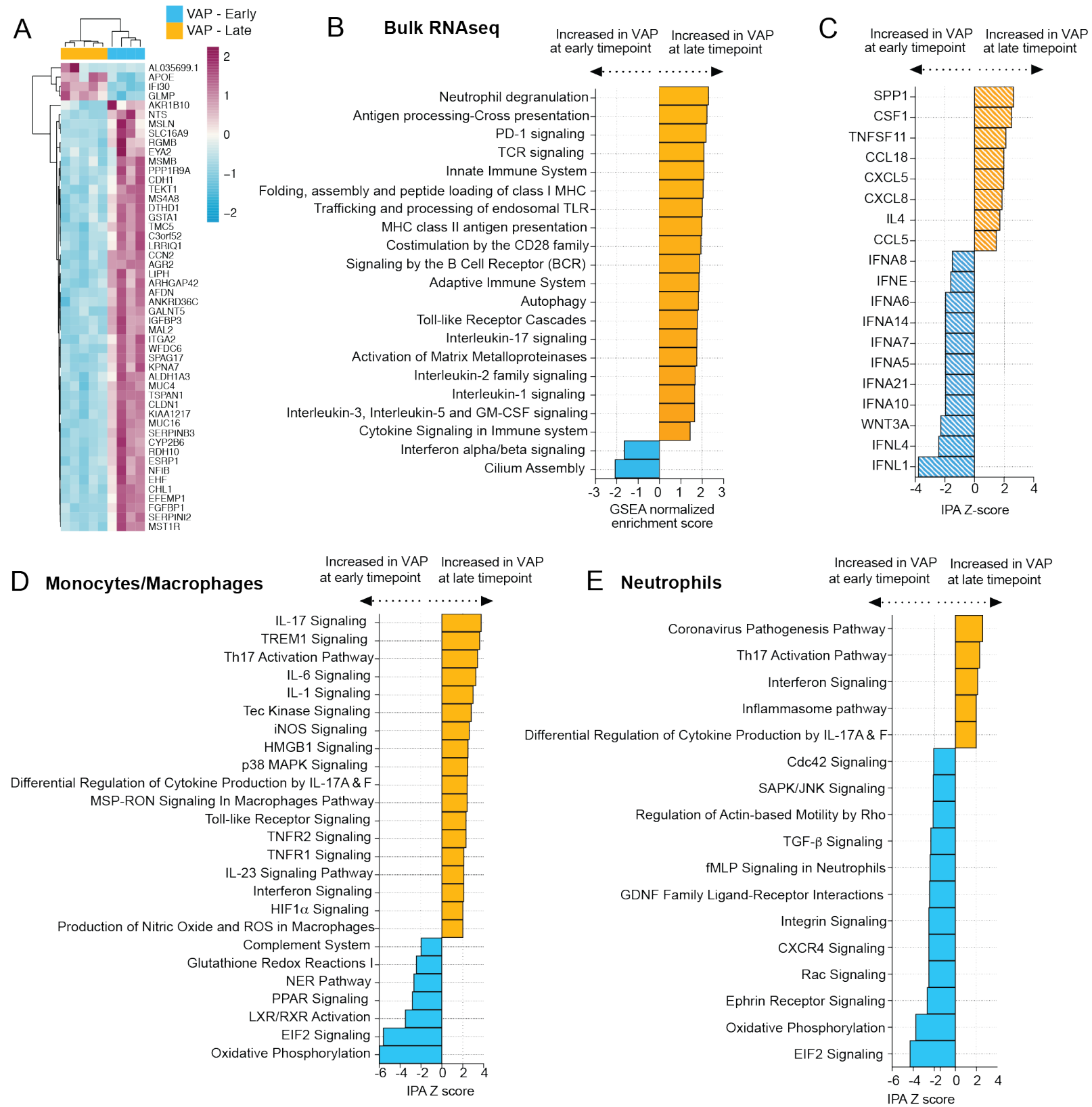

Increased in VAP Increased in VAP at early timepoint at late timepoint

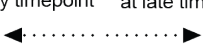

Coronavirus Pathogenesis Pathway

Th17 Activation Pathway

Interferon Signaling

Inflammasome pathway

Differential Regulation of Cytokine Production by IL-17A \& F

Cdc42 Signaling

SAPK/JNK Signaling

Regulation of Actin-based Motility by Rho-

TGF- $\beta$ Signaling

fMLP Signaling in Neutrophils

GDNF Family Ligand-Receptor Interactions

Integrin Signaling

CXCR4 Signaling

Rac Signaling

Ephrin Receptor Signaling

Oxidative Phosphorylation

EIF2 Signaling

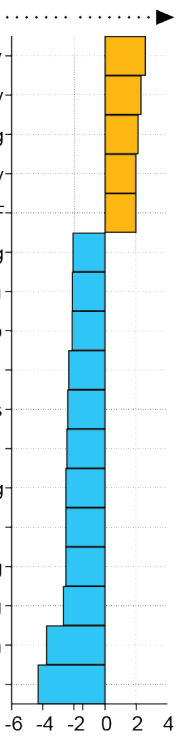

IPA Z score

F

\section{Innate Immune System}

$p=0.0020$

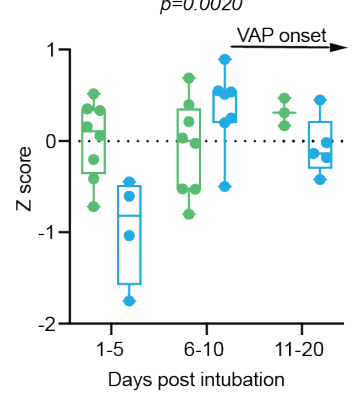

G

Neutrophil Degranulation

$p<0.001$

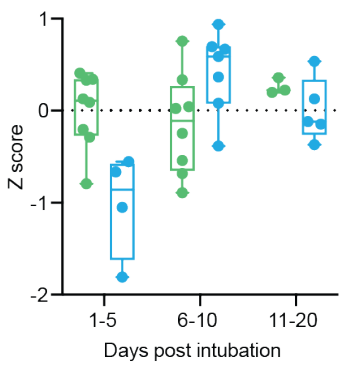

$\mathrm{H}$

Cytokine Signaling

$p=0.024$

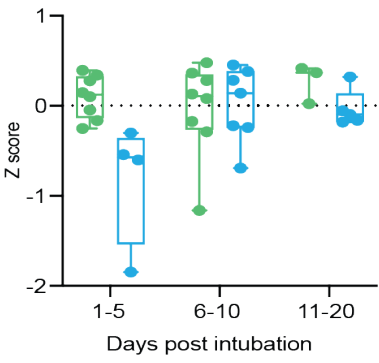

I

Adaptive Immune System $p=0.013$

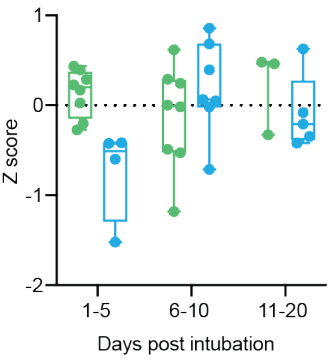


Figure 5: Temporal dynamics of the host response to VAP

A) Heatmap of the top 50 differentially expressed genes by adjusted P-value between COVID-19 patients who developed VAP at the "early" time-point (blue) versus the "late" time-point (yellow) from bulk RNA-seq. B) Gene set enrichment analysis (GSEA) based on differential gene expression of VAP patients at the "early" vs "late" time-point from bulk RNA-seq. GSEA results were considered significant with an adjusted P-value $<0.05$. C) Ingenuity Pathway Analysis (IPA) of upstream cytokines based on differential gene expression analyses of VAP patients at the "early" vs "late" time-point from bulk RNA-seq. IPA results were considered significant with a Zscore absolute value $>2$ and overlap P-value $<0.05$. (D-E) Ingenuity Pathway Analysis (IPA) of key canonical pathways based on differential gene expression analysis in monocytes and macrophages (D) or neutrophils (E) from scRNA-seq of patients who develop VAP versus those who do not, with adjusted $p$-values $<0.05$. Only significant pathways (IPA Z-score of $>2$ or $<-2$ and overlap $p$-value $<0.05$ ) are shown. All pathways and cytokines are shown in Supplementary data files 2, 3, 5, and 6. (F-I) Longitudinal analysis of selected pathway expression in VAP (blue) versus No-VAP (green) patients from bulk RNA-seq samples taken from time of intubation to onset of VAP for all patients. Pathway Z-scores were calculated by averaging Z-scores for the top 20 leading edge genes of each pathway, determined by the results of GSEA comparing VAP versus No-VAP patients at the "early" time-point. Multiple Z-scores per patient at a given time interval were averaged so that each patient corresponds to one datapoint at each interval. Samples from day 21+ after intubation are not shown due to a lack of these later time-points in the No-VAP group. VAP onset in these patients ranged from 10-39 days post intubation. Selected pathways are innate immune system $(F)$, neutrophil degranulation $(G)$, cytokine signaling $(H)$, and adaptive immune system (I). Box plots represent the median and range. Statistical significance was determined by two-way ANOVA, and interaction p-values are shown. 
Figure 6
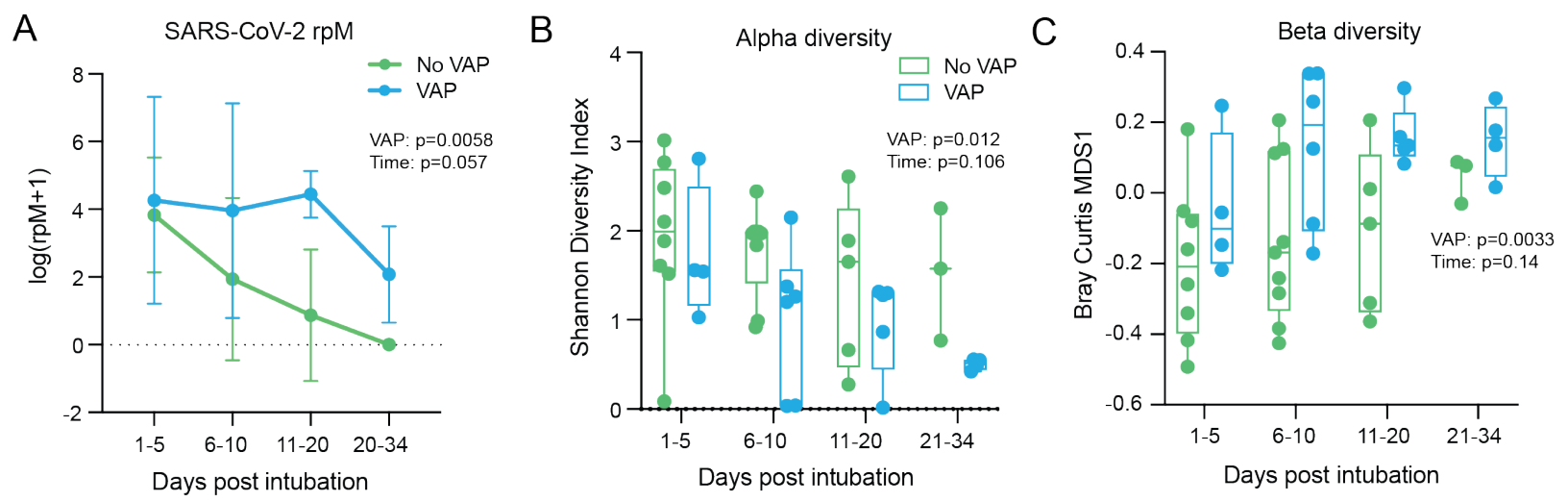

Figure 6: Lung microbiome community collapse precedes VAP in COVID-19 patients.

(A) SARS-CoV-2 viral load (reads per million sequenced, rpM) over time by days since intubation in patients who develop VAP vs those who do not. For plotting purposes, log $(r p M+1)$ was used to avoid negative values. Lung microbiome (B) bacterial diversity (Shannon's Index) and (C) $\beta$-diversity (Bray Curtis Index, NMDS scaling) in COVID-19 patients with relation to VAP development over time by days since intubation. Box plots represent the median and range (AC). Statistical significance was determined by two-way ANOVA. P-values $<0.05$ were considered significant.

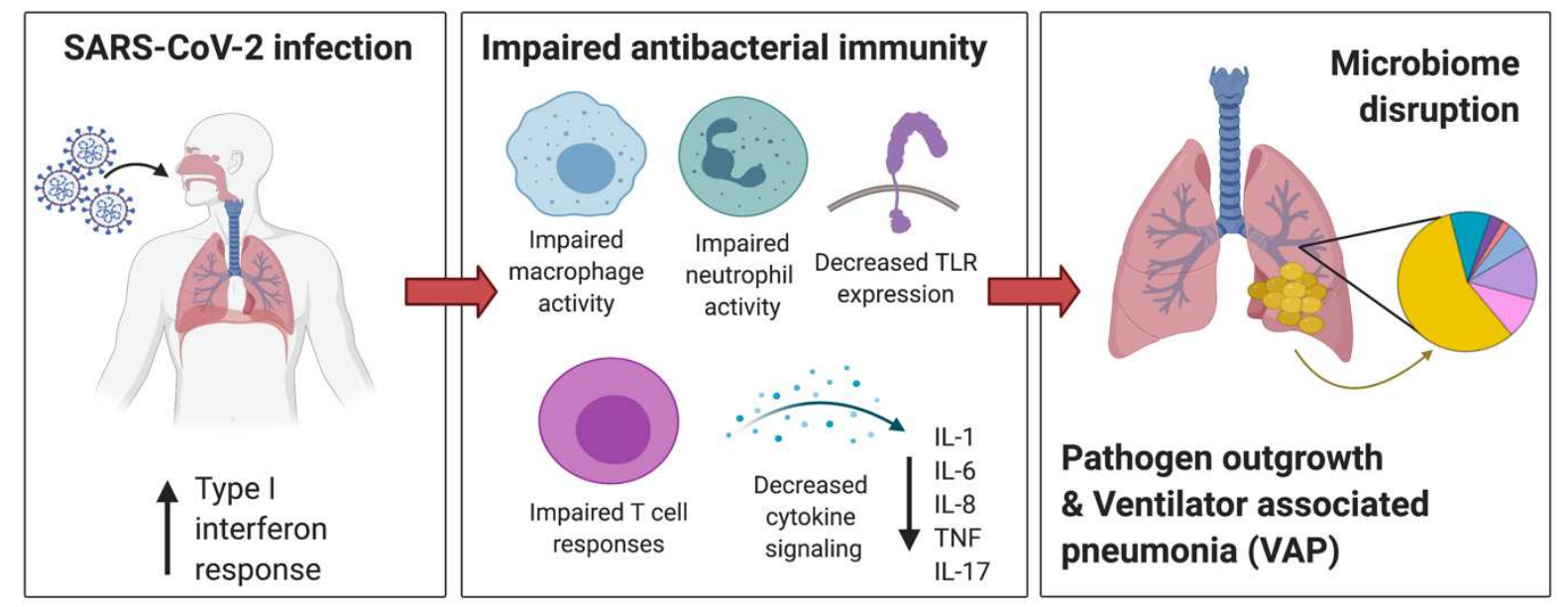

Figure 7: Mechanistic hypothesis of secondary bacterial pneumonia susceptibility in patients with COVID-19.

Individual immune responses to SARS-CoV-2 infection drive a restructuring of the microbial community and increase susceptibility to VAP. Those predisposed to VAP have increased type I interferon responses and dysregulated antibacterial immune signaling characterized by impaired macrophage, neutrophil and T cell activity, decreased TLR signaling and impaired activation of key cytokines important for pathogen defense including IL-1, IL-6, IL-8, TNF, and IL-17. This state of suppressed immunity disrupts the lower respiratory tract microbiome, predisposing to outgrowth of bacterial pathogens and VAP. 


\section{Figures}

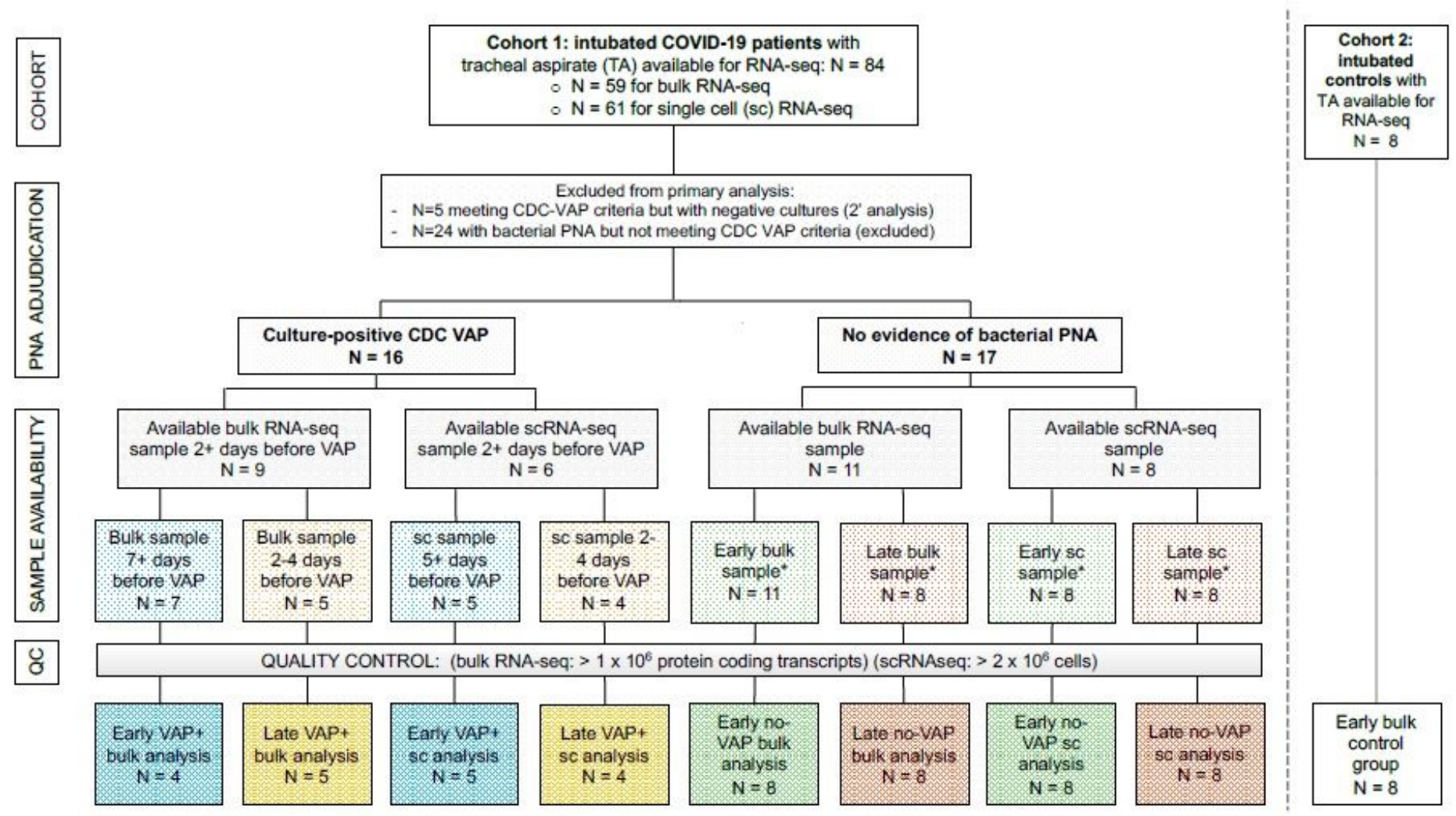

Figure 1

Study flowchart. Two patient cohorts were studied. Cohort 1 consisted of COVID-19 patients from the COVID Multiphenotyping for Effective Therapies (COMET) / Immunophenotyping Assessment in a COVID-19 Cohort (IMPACC) studies (described in Methods). Cohort 2 consisted of critically ill intubated control patients from a prior prospective cohort study led by our research group 18. The "early" samples were the first available tracheal aspirate specimens after intubation. For COVID- 19 patients who developed VAP, the "late" samples were obtained a median of two days before VAP onset. Timing of sample collection with respect to VAP versus No-VAP groups was matched at "early" and "late" time points. Controls included eight critically ill, mechanically ventilated patients without LRTI. All COVID-19 patients included in the primary bulk analysis were also included in the longitudinal host expression and microbiome analyses. Abbreviations: VAP=ventilator-associated pneumonia; TA=tracheal aspirate; $\mathrm{QC}=$ quality control; sc or scRNAseq= single cell RNA sequencing; $P N A=$ pneumonia; $C D C=$ United States Centers for Disease Control and Prevention. 

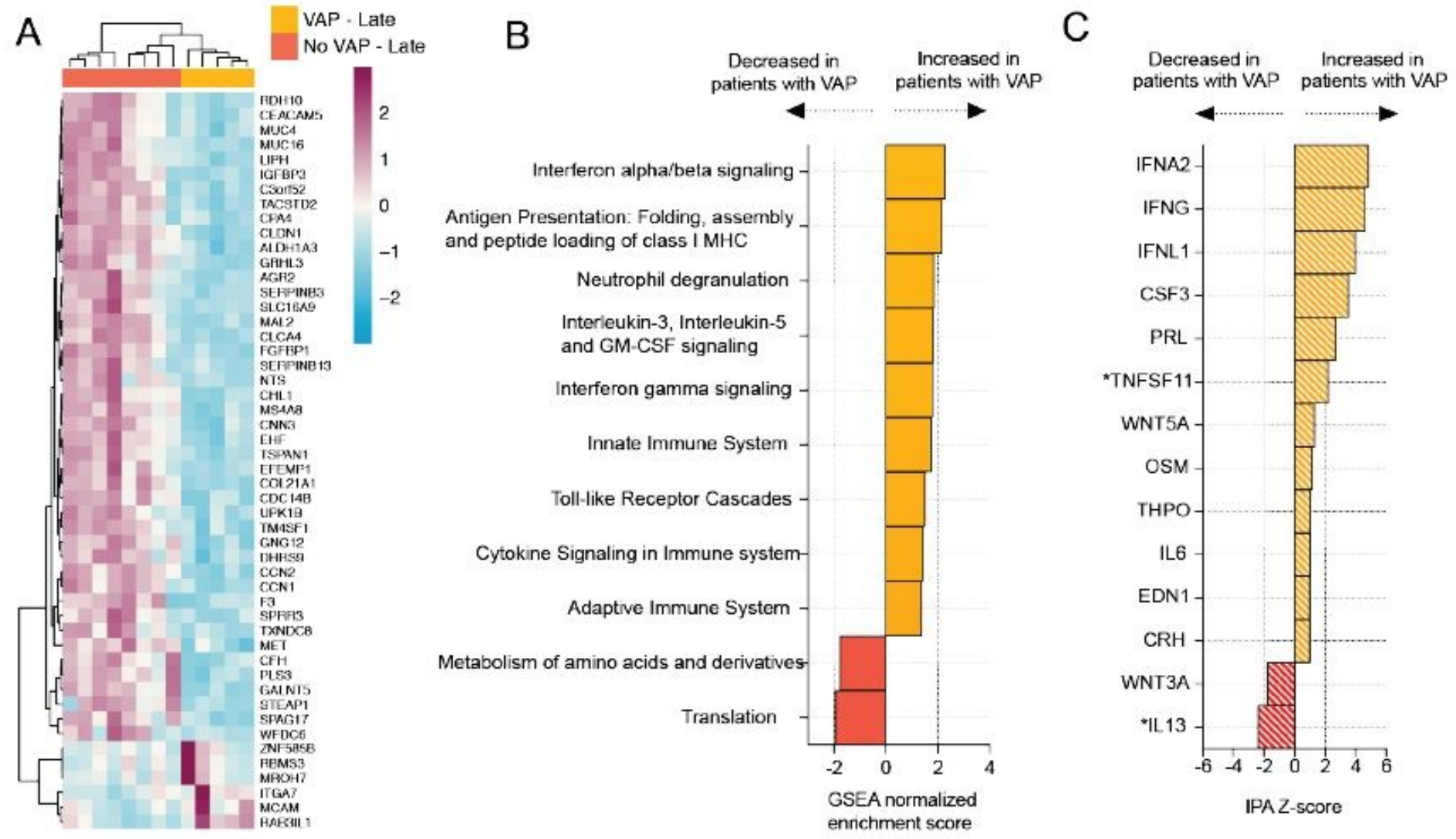

\section{Figure 2}

COVID-19 VAP is associated with a lower respiratory tract transcriptional signature of bacterial infection 2 days before VAP onset. A) Heatmap of the top 50 differentially expressed genes by adjusted P-value between COVID-19 patients who developed VAP (yellow) versus those who did not (red) at the "late" timepoint, 2 days before the onset of VAP, from bulk RNA-seq. B) Gene set enrichment analysis (GSEA) at the "late" time-point based on differential gene expression analyses. GSEA results were considered significant with an adjusted P-value $<0.05$. C) Ingenuity Pathway Analysis (IPA) of upstream cytokines at the "late" time-point based on differential gene expression analyses. IPA results were considered significant with a Z-score absolute value $>2$ and overlap P-value $<0.05$. *Denotes cytokines with an overlap P-value $<0.1$. All pathways and cytokines are shown in Supplementary data files 2 and 3. 

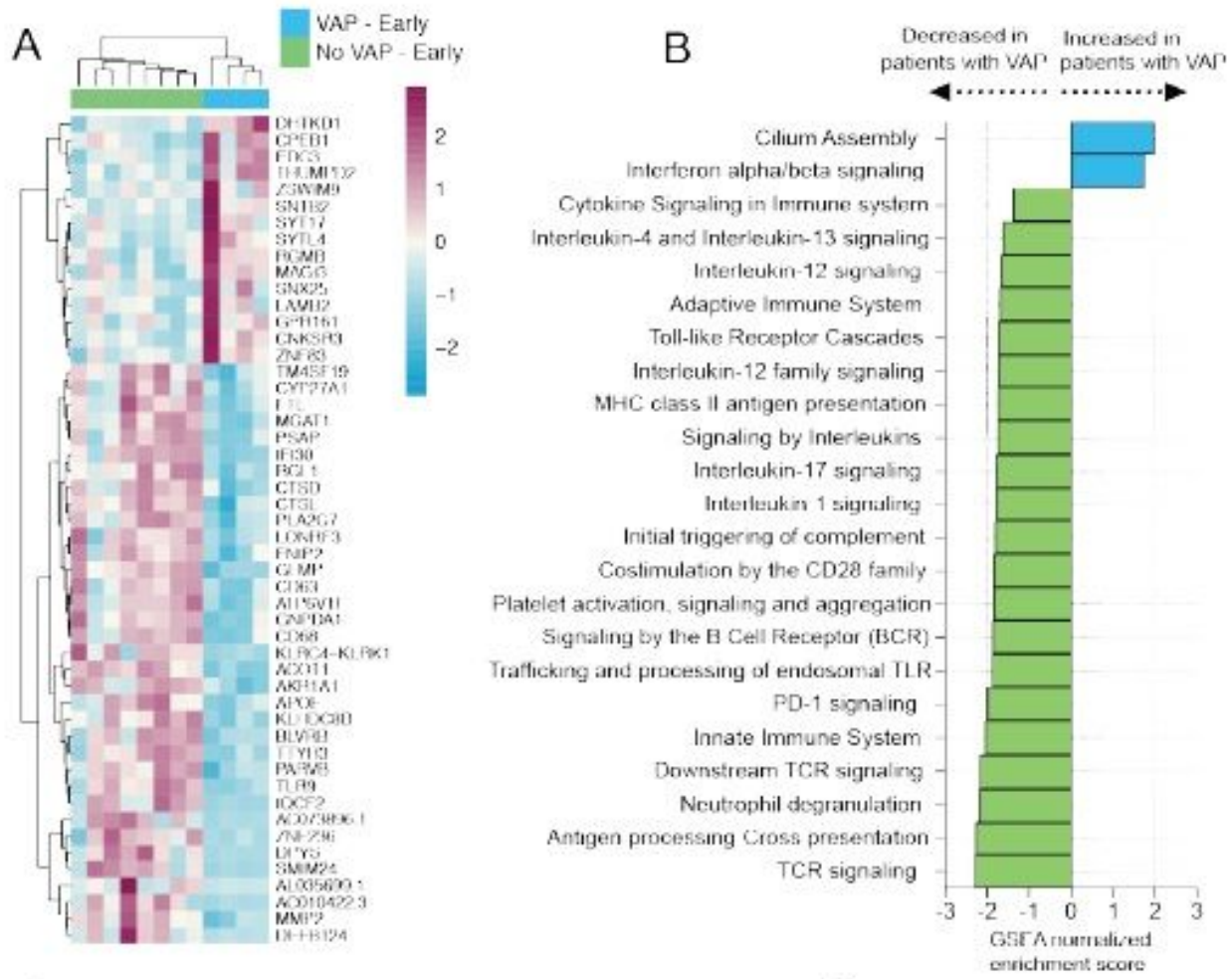

C
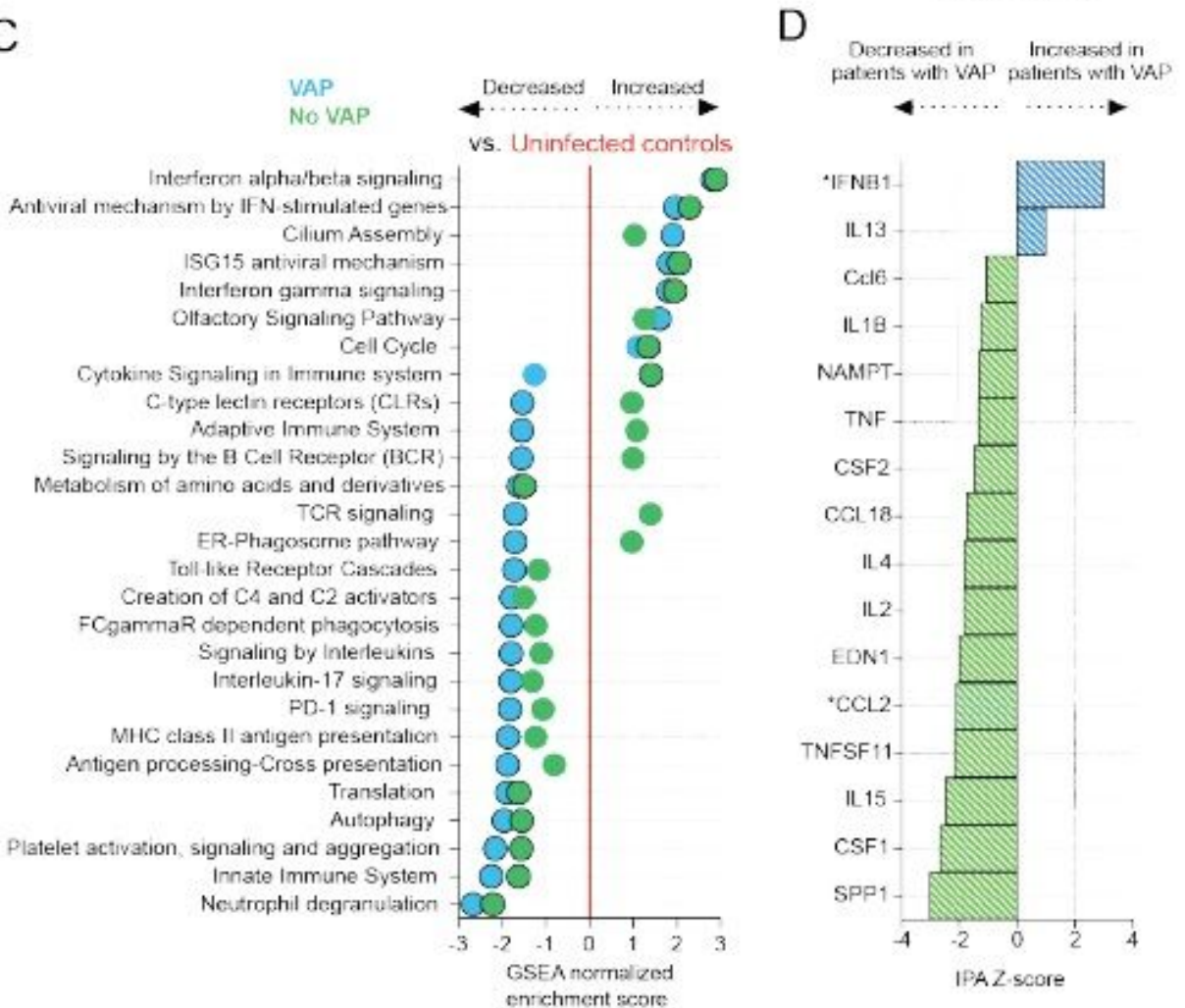

Figure 3

COVID-19 patients who develop VAP have attenuated immune signaling in the lower respiratory tract two weeks before onset of secondary bacterial pneumonia. A) Heatmap of the top 50 differentially expressed genes by adjusted P-value between COVID-19 patients who developed VAP (blue) versus those who did not (green) at the "early" time-point from bulk RNA-seq. B) Gene set enrichment analysis at the "early" time-point based on differential gene expression analyses. GSEA results were considered significant with 
an adjusted P-value $<0.05$. C) Expression of GSEA pathways at the "early" time-point with respect to a baseline of uninfected, intubated controls. Pathways were selected from the GSEA results if they had an adjusted P-value $<0.05$ in at least one of the comparisons (VAP vs controls or No-VAP vs controls).

Pathways with an adjusted P-value $<0.05$ when compared to controls are indicated by circles with a black outline. D) Ingenuity Pathway Analysis (IPA) of upstream cytokines at the "early" time-point based on differential gene expression analyses. IPA results were considered significant with a Z-score absolute value $>2$ and overlap P-value $<0.05$. *Denotes cytokines with an overlap P-value $<0.1$. All pathways and cytokines are shown in Supplementary data files 2 and 3. 


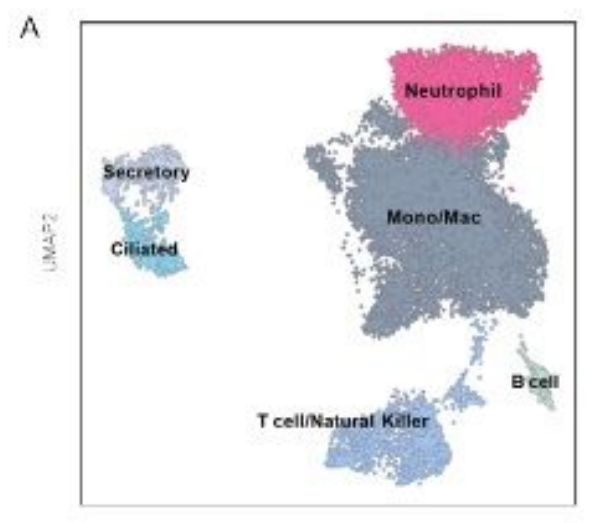

B
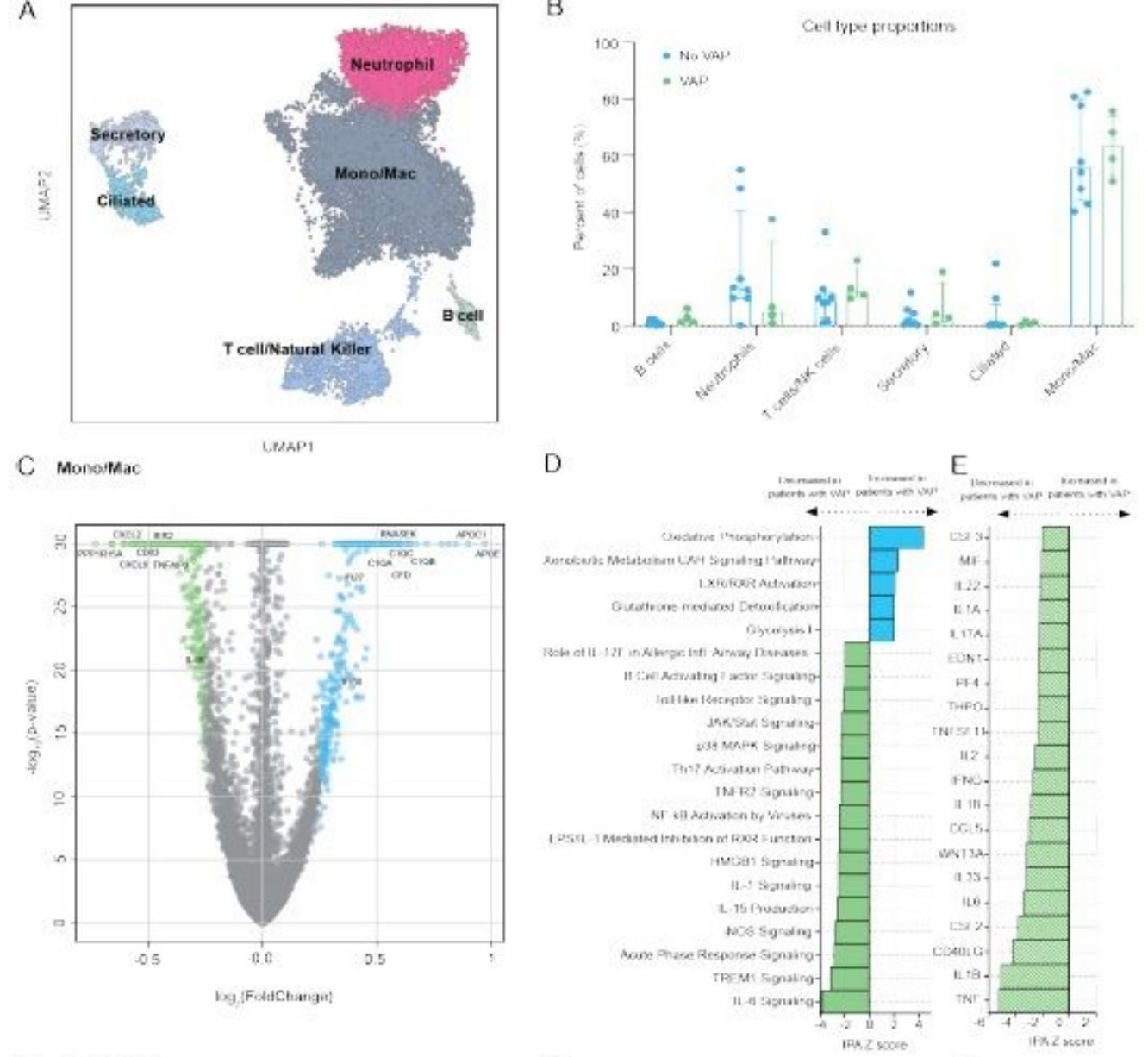

F Neutraphils

G
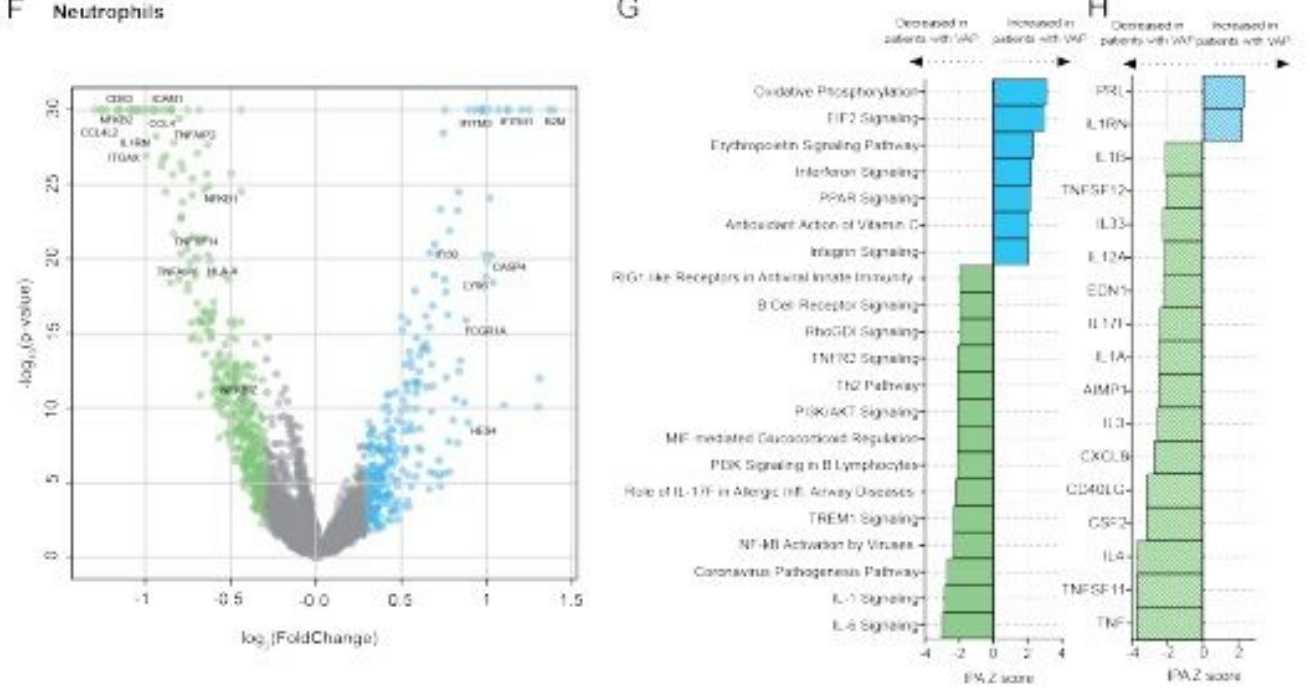

\section{Figure 4}

scRNA-seq demonstrates that COVID-19 VAP is associated with early impaired anti-bacterial immune signaling in lower respiratory tract monocytes, macrophages and neutrophils. A) UMAP of single cell RNA-seq data from patients that do or do not develop VAP at the "early" time-point, annotated by cell type. B) Cell type proportions in single cell RNA-seq from VAP and No-VAP patients at the "early" timepoint. Bars represent the median with IQR. Statistical significance was determined by Mann-Whitney 
tests. None of the cell types were significantly different with a $p$-value $<0.05$. The $p$-values for each cell type are as follows: B cells: 0.073; Neutrophils: $0.28 ;$ T/NK cells: 0.21 ; Secretory: 0.46 ; Ciliated: 0.94 , and Mono/Mac: 0.81 . C) Volcano plot displaying the differentially expressed genes between VAP and No-VAP patients in monocytes and macrophages. D) Ingenuity Pathway Analysis (IPA) of key canonical pathways and upstream cytokines based on differential gene expression analysis in monocytes and macrophages of patients who develop VAP versus those who do not, with adjusted $p$-values $<0.05$. Only significant pathways (IPA Z-score of $>2$ or $<-2$ and overlap p-value $<0.05$ ) are shown. E) Volcano plot displaying the differentially expressed genes between VAP and No-VAP patients in neutrophils. F) IPA of canonical pathways and upstream cytokines based on differential gene expression analysis in neutrophils of patients who develop VAP versus those who do not, with adjusted $p$-values $<0.05$. Only significant pathways (IPA Z-score of $>2$ or $<-2$ and overlap p-value $<0.05$ ) are shown. All pathways and cytokines are shown in Supplementary data files 5 and 6 . 

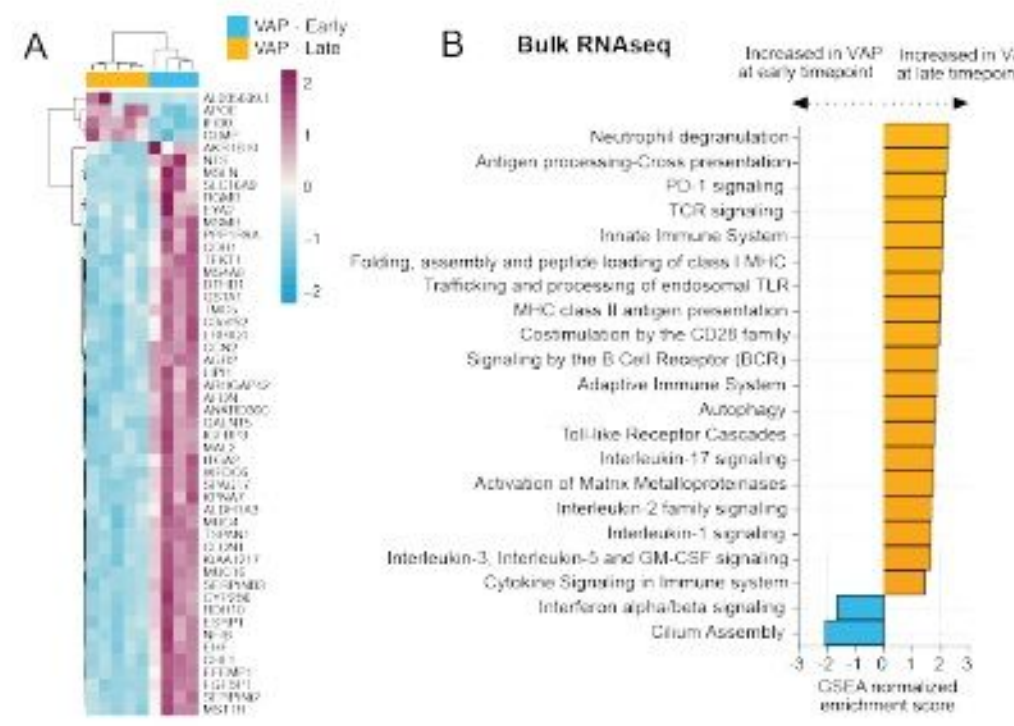

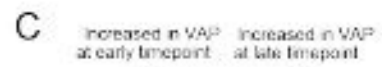
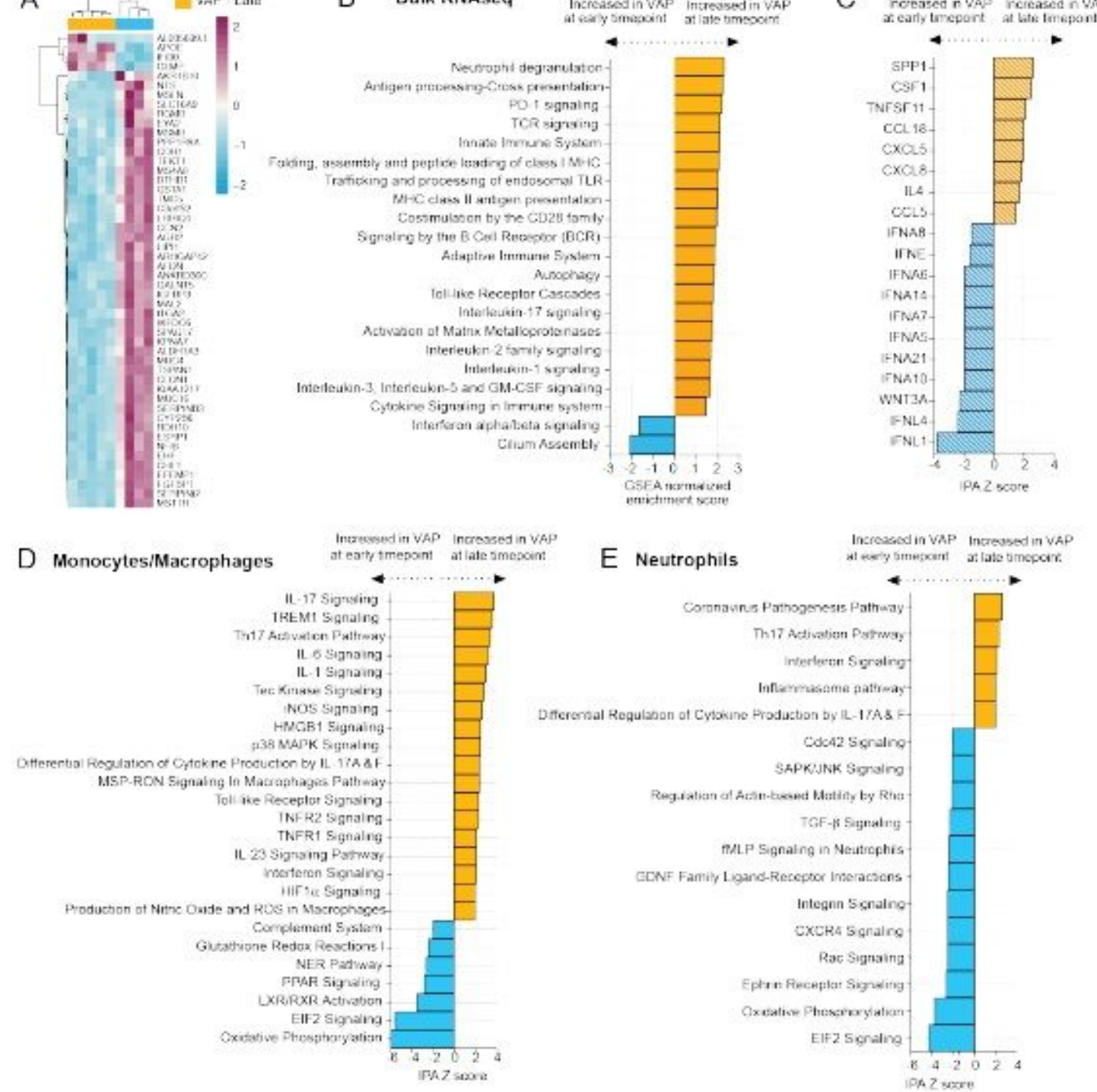

Increacod in VAP Increased in VAP

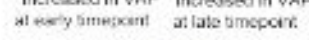

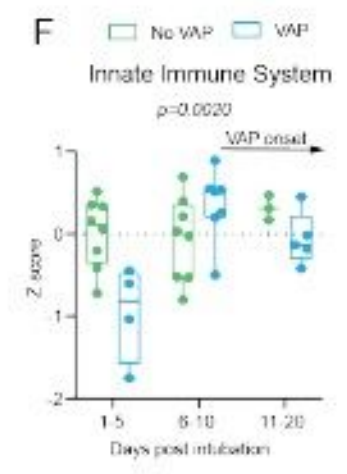

G

Neutrophil Degranulation

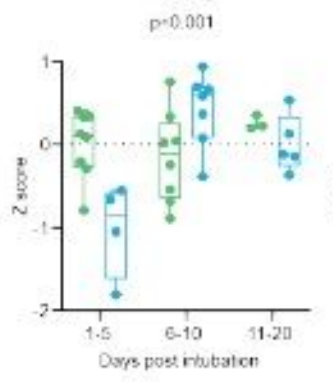

$\mathrm{H}$

Cytokine Signaling

$p-0.024$

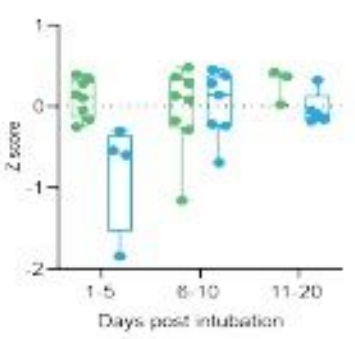

I

Adaptive Immune System $p=0013$

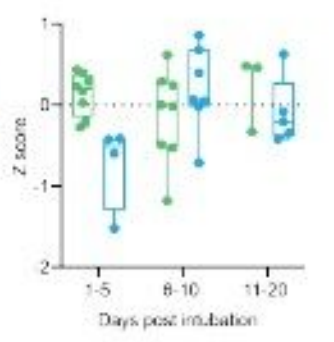

\section{Figure 5}

Temporal dynamics of the host response to VAP A) Heatmap of the top 50 differentially expressed genes by adjusted P-value between COVID-19 patients who developed VAP at the "early" time-point (blue) versus the "late" time-point (yellow) from bulk RNA-seq. B) Gene set enrichment analysis (GSEA) based on differential gene expression of VAP patients at the "early" vs "late" time-point from bulk RNA-seq. GSEA results were considered significant with an adjusted P-value $<0.05 . \mathrm{C}$ ) Ingenuity Pathway Analysis (IPA) 
of upstream cytokines based on differential gene expression analyses of VAP patients at the "early" vs "late" time-point from bulk RNA-seq. IPA results were considered significant with a Zscore absolute value $>2$ and overlap P-value <0.05. (D-E) Ingenuity Pathway Analysis (IPA) of key canonical pathways based on differential gene expression analysis in monocytes and macrophages (D) or neutrophils (E) from scRNA-seq of patients who develop VAP versus those who do not, with adjusted $p$-values $<0.05$. Only significant pathways (IPA Z-score of $>2$ or $<-2$ and overlap p-value $<0.05$ ) are shown. All pathways and cytokines are shown in Supplementary data files 2, 3, 5, and 6. (F-I) Longitudinal analysis of selected pathway expression in VAP (blue) versus No-VAP (green) patients from bulk RNA-seq samples taken from time of intubation to onset of VAP for all patients. Pathway Z-scores were calculated by averaging Zscores for the top 20 leading edge genes of each pathway, determined by the results of GSEA comparing VAP versus No-VAP patients at the "early" time-point. Multiple Z-scores per patient at a given time interval were averaged so that each patient corresponds to one datapoint at each interval. Samples from day 21+ after intubation are not shown due to a lack of these later time-points in the No-VAP group. VAP onset in these patients ranged from 10-39 days post intubation. Selected pathways are innate immune system (F), neutrophil degranulation $(\mathrm{G})$, cytokine signaling $(\mathrm{H})$, and adaptive immune system $(\mathrm{I})$. Box plots represent the median and range. Statistical significance was determined by two-way ANOVA, and interaction $\mathrm{p}$ values are shown.

A

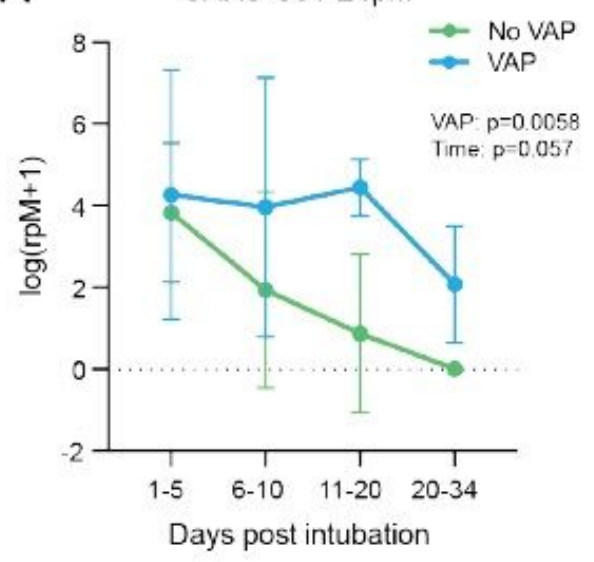

B

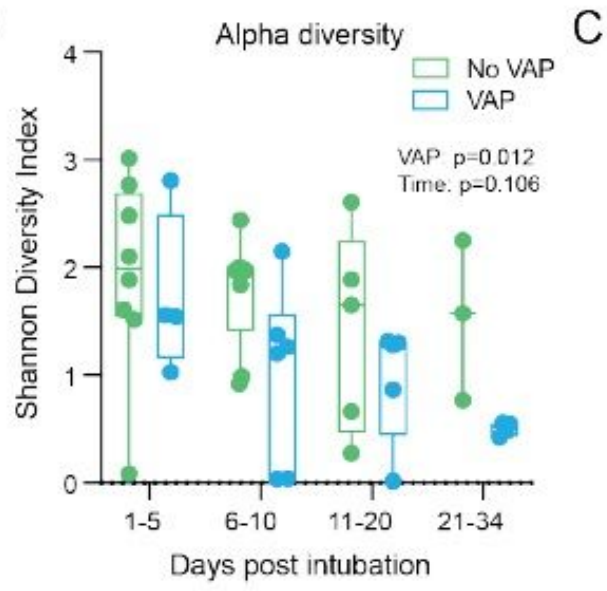

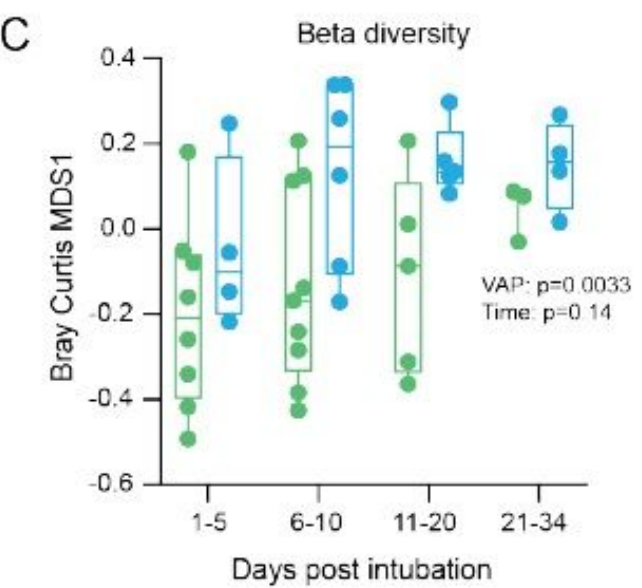

\section{Figure 6}

Lung microbiome community collapse precedes VAP in COVID-19 patients. (A) SARS-CoV-2 viral load (reads per million sequenced, rpM) over time by days since intubation in patients who develop VAP vs those who do not. For plotting purposes, $\log (\mathrm{rpM}+1)$ was used to avoid negative values. Lung microbiome (B) bacterial diversity (Shannon's Index) and (C) b-diversity (Bray Curtis Index, NMDS scaling) in COVID-19 patients with relation to VAP development over time by days since intubation. Box plots represent the median and range (AC). Statistical significance was determined by two-way ANOVA. Pvalues $<0.05$ were considered significant. 


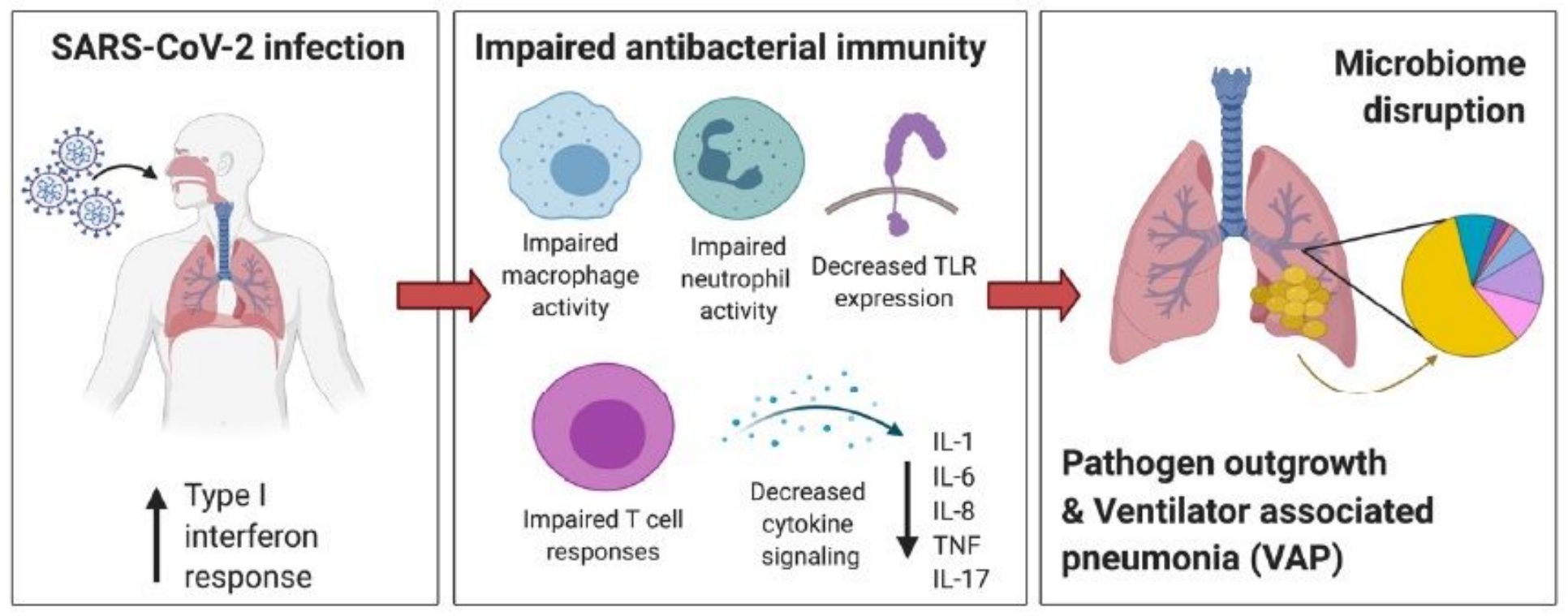

\section{Figure 7}

Mechanistic hypothesis of secondary bacterial pneumonia susceptibility in patients with COVID-19. Individual immune responses to SARS-CoV-2 infection drive a restructuring of the microbial community and increase susceptibility to VAP. Those predisposed to VAP have increased type I interferon responses and dysregulated antibacterial immune signaling characterized by impaired macrophage, neutrophil and T cell activity, decreased TLR signaling and impaired activation of key cytokines important for pathogen defense including IL-1, IL-6, IL-8, TNF, and IL-17. This state of suppressed immunity disrupts the lower respiratory tract microbiome, predisposing to outgrowth of bacterial pathogens and VAP.

\section{Supplementary Files}

This is a list of supplementary files associated with this preprint. Click to download.

- SupplementaryAppendixCOMETConsortiumMemberlist.xlsx

- SupplementaryMaterialsNC3.31.2021.pdf

- DATAS1BulkRNAseqDEgenes.xIsx

- DATAS2BulkRNAseqFGSEA.xIsx

- DATAS3BulkRNAseqIPAUpstreamRegCytokines.xlsx

- DATAS4singlecelIRNAseqDEgenes.xIsx

- DATAS5scRNAseqIPACannonicalPath.xlsx

- DATAS6scRNAseqIPAUpstreamRegCytokines.xIsx

- DATAS7PatientMetadata.xIsx

- nrreportingsummaryNCOMMS2112534.pdf

- nrsoftwarepolicyNCOMMS2112534.pdf 